\title{
Apicidin biosynthesis is linked to accessory chromosomes in Fusarium poae isolates
}

\author{
Thomas E. Witte ${ }^{1,2}$, Linda J. Harris ${ }^{1}$, Hai D. T. Nguyen ${ }^{1}$, Anne Hermans ${ }^{1}$, Anne Johnston', Amanda Sproule, \\ Jeremy R. Dettman ${ }^{1}$, Christopher N. Boddy ${ }^{2}$ and David P. Overy ${ }^{1 *}$
}

\begin{abstract}
Background: Fusarium head blight is a disease of global concern that reduces crop yields and renders grains unfit for consumption due to mycotoxin contamination. Fusarium poae is frequently associated with cereal crops showing symptoms of Fusarium head blight. While previous studies have shown F. poae isolates produce a range of known mycotoxins, including type A and B trichothecenes, fusarins and beauvericin, genomic analysis suggests that this species may have lineage-specific accessory chromosomes with secondary metabolite biosynthetic gene clusters awaiting description.
\end{abstract}

Methods: We examined the biosynthetic potential of $38 \mathrm{~F}$. poae isolates from Eastern Canada using a combination of long-read and short-read genome sequencing and untargeted, high resolution mass spectrometry metabolome analysis of extracts from isolates cultured in multiple media conditions.

Results: A high-quality assembly of isolate DAOMC 252244 (Fp157) contained four core chromosomes as well as seven additional contigs with traits associated with accessory chromosomes. One of the predicted accessory contigs harbours a functional biosynthetic gene cluster containing homologs of all genes associated with the production of apicidins. Metabolomic and genomic analyses confirm apicidins are produced in 4 of the 38 isolates investigated and genomic PCR screening detected the apicidin synthetase gene APS1 in approximately $7 \%$ of Eastern Canadian isolates surveyed.

Conclusions: Apicidin biosynthesis is linked to isolate-specific putative accessory chromosomes in F. poae. The data produced here are an important resource for furthering our understanding of accessory chromosome evolution and the biosynthetic potential of F. poae.

Keywords: Fusarium poae, Metabolomics, Genomics, Secondary metabolites, Apicidin, Fungal plant pathogens, Mass spectrometry, Accessory chromosomes, Biosynthetic gene clusters

\section{Background}

Fusarium head blight (FHB) is an economically devastating cereal crop disease that reduces yields, contaminates grains with harmful mycotoxins, and represents a threat to global food security [1]. Surveys of FHB-symptomatic cereals often detect a complex of different Fusarium

\footnotetext{
* Correspondence: david.overy@canada.ca

'Agriculture and Agri-Food Canada, Ottawa Research and Development Centre, Ottawa, Canada

Full list of author information is available at the end of the article
}

species, including $F$. graminearum, $F$. avenaceum and $F$. poae [2-5]. While epidemic-level outbreaks of the disease on wheat and barley are attributed to aggressive isolates of $F$. graminearum, the routine detection of other close relatives in the F. sambucinum and F. incarnatumequisiti species complexes in FHB-infected plants implies an intricate disease model involving fungi with varying levels of pathogenicity. In a recent survey of Canadian wheat, barley and oats, F. poae was the Fusarium species most frequently isolated from FHB-

(c) Her Majesty the Queen in Right of Canada as represented by the Minister of Agriculture and Agri-Food. 2021 Open Access This article is licensed under a Creative Commons Attribution 4.0 International License, which permits use, sharing, adaptation, distribution and reproduction in any medium or format, as long as you give appropriate credit to the original author(s) and the source, provide a link to the Creative Commons licence, and indicate if changes were made. The images or other third party material in this article are included in the article's Creative Commons licence, unless indicated otherwise in a credit line to the material. If material is not included in the article's Creative Commons licence and your intended use is not permitted by statutory regulation or exceeds the permitted use, you will need to obtain permission directly from the copyright holder. To view a copy of this licence, visit http://creativecommons.org/licenses/by/4.0/. The Creative Commons Public Domain Dedication waiver (http://creativecommons.org/publicdomain/zero/1.0/) applies to the data made available in this article, unless otherwise stated in a credit line to the data. 
symptomatic oat samples and was as frequently isolated from barley as was $F$. graminearum [4]. The association of $F$. poae with FHB-damaged cereals is a global trend [5-7] that calls for investigation into fundamental aspects of the species' life cycle, population structure and biosynthetic potential.

F. poae can produce a diverse range of mycotoxins including a mixture of type $\mathrm{A}$ and type $\mathrm{B}$ trichothecenes [8]. Neosolaniol, diacetoxyscirpenol, monoacetoxyscirpenol, fusarenone- $X$, nivalenol and scirpentriol have all been detected from F. poae isolates [9], and the extent of their accumulation in cereal grains depends on the producing isolate, the grain type and the environmental context in which the plants are grown [10]. Trichothecene detection and characterization are a top priority for food safety analysts due to their deleterious effects as protein synthesis inhibitors and their alteration of membrane properties [11]. However, the toxigenic potential of $F$. poae isn't limited to trichothecenes. Beauvericin, enniatins and fusarins have also been detected from $F$. poae isolates grown in laboratory conditions and from FHB-damaged grain [9]. While none of these mycotoxins are monitored or regulated in Canada or Europe, in part due to insufficient studies of in vivo toxicity and lack of toxicokinetic data [12, 13], in vitro and in vivo studies suggest many of these molecules have genotoxic effects, have immunomodulating activity, and in some cases pose a reproductive health hazard to consumers [13]. Molecules such as enniatin and beauvericin have been labeled 'emerging mycotoxins' and may be placed under increased regulatory scrutiny in the future due to our evolving understanding of their in vivo bioactivity. Furthermore, the potential roles of these molecules (often termed 'secondary metabolites') in the context of plant invasion are poorly understood.

Technological advances in analytical chemistry now enable untargeted, simultaneous detection and identity prediction of complex secondary metabolite mixtures, providing an unprecedented view of fungal biosynthetic output. When coupled with high quality long-read genome sequencing tools, untargeted chemical profiling can be used to correlate metabolomes of individual fungal isolates to biosynthetic gene cluster (BGC) diversity within populations. BGCs are usually defined by their core, molecular scaffold-building enzymes, which include polyketide synthases (PKS), non-ribosomal peptide synthetases (NRPS), or terpene synthase/cyclases. Predicted clusters also include 'tailoring' enzymes such as oxidoreductases and methyl transferases, as well as transcription factors and transport-related genes. Genes within BGCs are presumed to be co-regulated during activation of the cluster - however the environmental or biological triggers for BGC activation are multifactorial. Pan-genomic analyses indicate Fusarium species, including F. poae, possess many undescribed 'cryptic' BGCs which may not be expressed under common in vitro culturing conditions [14-16]. The secondary metabolite products of these 'cryptic' BGCs may play critical roles in other contexts and may provide important clues as to how pathogens evolve. Developing a more complete picture of $F$. poae BGCs and associated secondary metabolites is therefore a key step in advancing our understanding of $F$. poae pathogenicity, particularly since many fungal plant pathogen genomes are dynamic and show the potential for rapid adaptation in response to plant defense mechanisms.

Profiling $F$. poae populations using untargeted metabolomics and long-read genomics is also prudent due to the recent discovery of accessory chromosomes (ACs) with secondary metabolite biosynthetic potential residing in a European isolate of the fungus [17]. ACs can be differentiated from core chromosomes by their non-Mendelian inheritance patterns, low gene density, high transposable element (TE) content and gene duplication frequencies. ACs have been studied extensively in other species of pathogenic fungi including Alternaria alternata [18], F. solani (formerly also known as Nectria haematococca) [19] and $F$. oxysporum f. sp. lycopersici [20]. In specific contexts relating to plant pathogenicity, ACs have been termed 'pathogenicity chromosomes' or 'conditionally dispensable chromosomes'. Although we recognize the use of the term $\mathrm{AC}$ departs from the equally valid term 'supernumerary chromosome' (previously used in conjunction with $F$. poae) [17], we believe that use of the term AC is more broadly consistent with fungal research literature as it relates ACs to 'accessory regions' (that share many of the key characteristics of ACs but reside on core chromosomes) [21]. ACs have been associated with novel plant host invasion in cases where they harbour fungal virulence factors (such as host specific toxins and effector proteins) and play an important role in plant pathogen niche invasion and adaptation [22]. The origins of ACs and associated genes are not always clear and may be diverse, including horizontal transfer between species/isolates or duplications and losses of core chromosome segments [23]. AC genetic content and the effects of disruptive TE transpositions between ACs and core chromosomes may generate novel genotypes in F. poae populations [17], promoting the evolution of increased virulence or niche invasion. Furthermore, F. poae isolates could theoretically produce AC-associated mycotoxins not currently screened by regulatory agencies in addition to the mycotoxins they are known to produce.

Previously published field surveys characterizing the occurrence of FHB in Eastern Canada from 2006 to 2017 , found $F$. poae to be associated with symptomatic wheat, barley and oat heads [4]. Herein, from a sample set of 184 Eastern Canadian isolates of $F$. poae, a subset of 38 isolates were chemically profiled using an 
untargeted UPLC-HRMS based metabolomics analysis, and the results compared to genome sequences in a 'multi-omics' approach focused on secondary metabolite detection, AC prediction and BGC analysis. This approach permits the correlation of predicted isolatespecific ACs encoding BGCs with chemical profile patterns. Finally, we present a high-quality genome assembly for isolate $F p 157$ which includes seven predicted accessory chromosome contigs totalling $5.2 \mathrm{Mb}$ or $8 \%$ of the total genome predicted size. This is a valuable resource to develop a greater understanding of the biosynthetic potential and structure of ACs in Fusarium.

\section{Results}

Isolate selection for genomic and metabolomic analysis

All isolates under investigation were initially identified morphologically and then confirmed as $F$. poae by TEF1- $\alpha$ gene sequence homology with other sequenced Fusaria at the Fusarium-ID website (http://isolate. fusariumdb.org/blast.php) [24]. From a culture collection of $184 \mathrm{~F}$. poae isolates, a subset of 38 isolates were selected for detailed genomic and metabolomic analysis (Additional file 1 contains a list of all isolates screened). The selection of the 38 isolates was based on: genetic variance associated with TEF1- $\alpha$ and trichothecene biosynthetic genes TRI1 and TRI8 (inferred phylogenetic tree is in Additional file 2); diverse metabolomic signatures from ultra-high performance liquid chromatography coupled to high resolution mass spectrometry (UPLC-HRMS) data of extracts from isolates grown on YES media; and variation in host crop and geographical origin. TRI1 and TRI8 were chosen due to previous analyses which showed variations in these genes led to alternate trichothecene modifications [25-27] and higher sequence divergence observed within previously genome sequenced F. poae isolates [17].

Genome assembly using Illumina sequence data of the 38 chosen isolates produced a median of 1375 scaffolds per genome and a mean genome length of $39.25 \mathrm{Mb}$. A summary of Illumina genome assembly statistics can be found in Additional file 3. To evaluate the completeness of the assemblies we identified BUSCO (Benchmarked Universal Single Copy Orthologs) [28] gene analysis using the Hypocreales_odb10 database, which revealed all genomes were over $97 \%$ complete, with the exception of one poor quality genome $(F p 030 ; 80.7 \%)$, and one failed sequencing run ( $F p 029$, not included in genomic analysis).

\section{A high-quality genome for $F p 157$ confirmed the secondary metabolite biosynthetic potential in $F$. poae and the presence of accessory chromosome-associated sequences}

$F$. poae isolate $F p 157$ was selected for long-read sequencing using the Oxford Nanopore platform (340,123 filtered reads, mean length of $21,465 \mathrm{bp}$ and mean qscore of 10.24). We generated a high quality genome (Fig. 1) with four core chromosomes exhibiting strong macrosynteny to the previously genome sequenced Belgian F. poae isolate 2516 [17] as well as F. graminearum isolate PH1 [29]. Additional file 4 contains genome assembly statistics for $F p 157$, and Additional file 5 contains LASTZ [30] dot plots comparing core chromosome synteny between Fp157, F. poae 2516 and F. graminearum PH1. A total of 14,114 genes were predicted, with two additional genes manually annotated based on blastn matches to biosynthetic genes (FpPKS2 and FpNRPS4, discussed in text). Core chromosomes Chr1 and Chr2 represent 'telomere to telomere' sequences with putative centromeric regions and telomeres comparable to the length, position and GC content of those described from $F$. graminearum $\mathrm{PH} 1$ [29]. Centromeres consist of approximately 50Kb-long regions averaging 15\% GC content, and telomeres show canonical 'TTAGGG' repeats followed by a 1500 bp region averaging $37 \% \mathrm{GC}$ content. Chr4 has telomeric repeats at the $5^{\prime}$ end, whereas the $3^{\prime}$ end encodes predicted rDNA repeats, also congruent with $F$. graminearum PH1. Chr3 is missing a telomeric sequence at the $5^{\prime}$ end and when compared to isolate 2516, subtelomeric regions appear inverted and rearranged. Lastly, mapping of the raw Nanopore and Illumina reads (data not shown) did not support the presence in $F p 157$ of the approximately $1 \mathrm{Mb}$ inversion detected in Chr1 of isolate 2516. This inversion is also not observed in Chr1 of $F$. graminearum PH1 (See Additional file 5 for LASTZ comparisons) [17].

In addition to the core chromosomes, 9 contigs were assembled in the $F p 157$ genome ranging in size from $100,057 \mathrm{bp}$ to $1,877,593 \mathrm{bp}$. Contig_5 is 140,862 bp long, representing the mitochondrial genome, and was not annotated. Contig_8 is 122,757 bp of rDNA repeats and is virtually identical to the $60 \mathrm{~Kb}$ of rDNA repeats associated with the $3^{\prime}$ end of Chr4. The remaining seven contigs cumulatively total $5,205,433 \mathrm{bp}$ and are presumed to represent $\mathrm{AC}$ sequences based on a number of factors. First, predicted ACs have genetic content that is congruent with the published content of predicted ACs in Belgian $F$. poae isolate 2516 [17]. Second, there is a lack of macrosynteny to sister species $F$. graminearum $\mathrm{PH} 1$ core chromosome sequences. Third, contigs are predicted to be less affected by repeat-induced point mutations (RIP, predicted by sliding-window dinucleotide frequency analysis [31]), when compared to core chromosomes, a pattern also congruent with $F$. poae 2516 ACs. Fourth, the contigs show elevated repetitive element content compared to core chromosomes, as seen in many confirmed fungal ACs [32]. Finally, the contigs show lower predicted gene densities when compared to core 


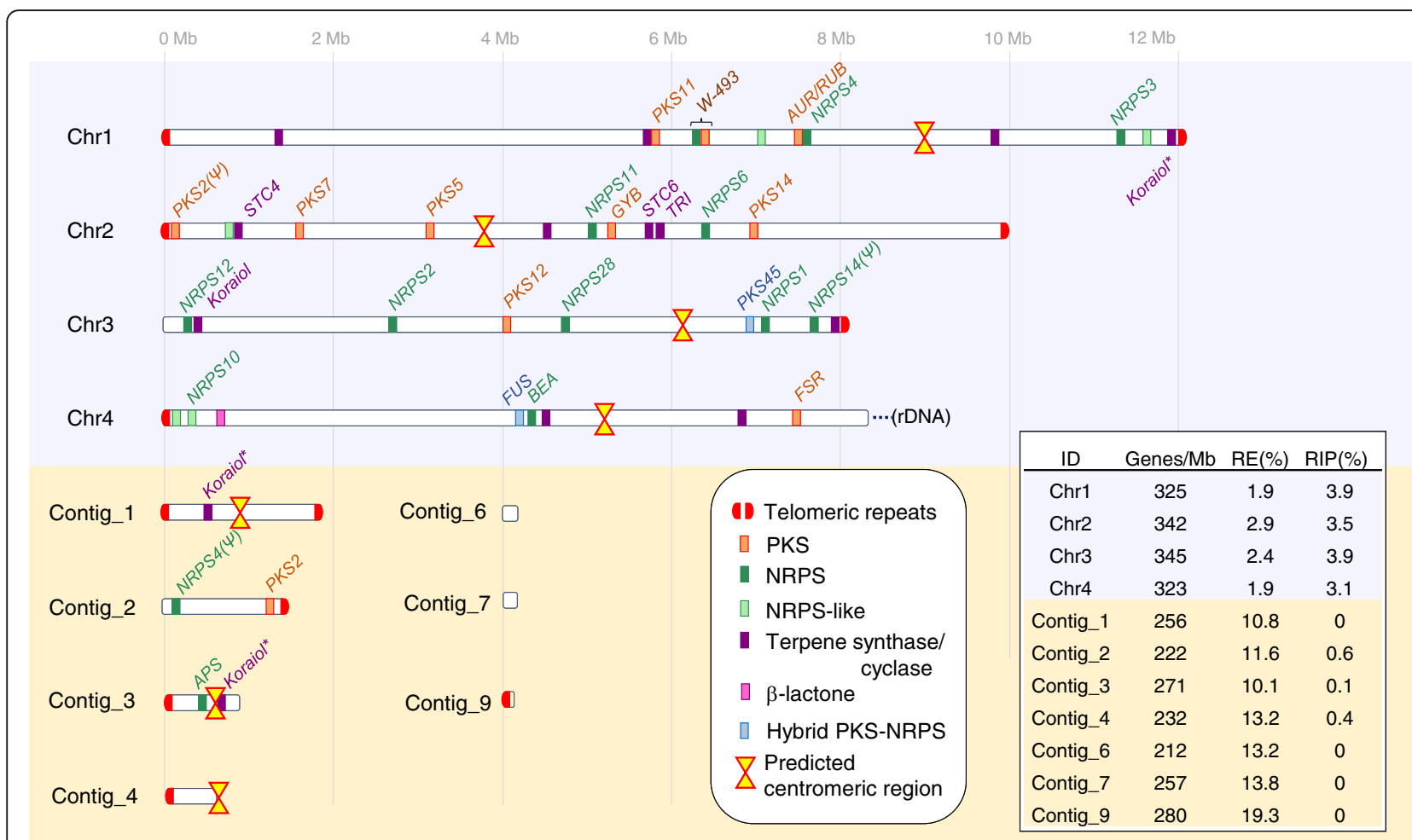

Fig. 1 Genome assembly of Fusarium poae isolate Fp157 with predicted biosynthetic gene clusters (BGCs), centromeric regions and telomeric repeats overlaid. BGC sizes are not to scale. Asterisks indicate duplicated koraiol synthases with $>98 \% \mathrm{nt} I \mathrm{D}$. Annotations above BGCs refer to associated synthase/synthetase clades, or associated mycotoxin products where known. RE indicates repeat element contents expressed as a percentage of each chromosome or contig length calculated independently of the rest of the genome (repeat content attributable to duplications between chromosomes was not calculated). Similarly, evidence of repeat-induced point mutation (RIP) was calculated independently per sequence and is expressed as the percent of each sequence predicted to be RIP-affected (evidenced by calculation of low GC-content compared to average dinucleotide frequencies). Predicted centromeres and telomeres were removed from all sequences prior to RIP analysis

chromosomes, another characteristic feature of ACs [20, 33] (Fig. 1).

Within the AC-associated contigs, there are three putative centromeric regions identified by similar size and GC content to core centromeres (contig_1, $52.7 \mathrm{~kb}$ averaging 14.5\% GC content; contig_3, $53.3 \mathrm{~kb}$ averaging $14.7 \%$ GC content; contig_4, $35.2 \mathrm{~kb}$ terminating at end of contig, averaging $15.6 \% \mathrm{GC}$ content). Additionally, six sets of telomeric repeats were identified and are all located on contig terminal regions. We therefore suggest there are three ACs in total, however further experimental verification including electrophoretic karyotyping is needed to confirm the number and size of the ACs, and to build telomere-to-telomere assemblies of their contents.

AntiSMASH 5.1 [34] analysis of the $F p 157$ genome predicted 43 discrete BGCs which encoded various core scaffold genes including 12 polyketide synthases (PKS), 13 terpene synthases, 10 non-ribosomal peptide synthetases (NRPS), 10 NRPS-like synthetases (usually a single NRPS module lacking a canonical domain) and 2 hybrid PKS-NRPS genes (Table 1). One cluster was predicted to be involved in the formation of $\beta$-lactones. Blastx comparison of NRPS adenylation domains, PKS ketosynthase domains and full-length PKS genes (all domains) to published databases of Fusarium-associated PKS and NRPS genes $[15,35]$ indicated all PKS and NRPS genes are orthologs of genes previously associated with Fusaria, and approximately half are associated with known products.

\section{Chemical phenotyping of $F$. poae isolates}

Chemical phenotypes for each of the 38 isolates grown in vitro were generated to visualize patterns in mass feature detection frequencies, and untargeted mass feature diversity. Mass features were obtained from UPLCHRMS profiles of extracts from isolates grown on five media conditions. Each media condition was chosen to diversify sources of nitrogen, sugars, salt stress and starvation stress. Media formulations used are detailed in Additional file 6. Mass feature intensities were converted to binary presence/absence for each media condition, and then averaged across all media conditions into a consensus phenotype for each isolate. Figure 2 
Table 1 Summary of predicted BGCs from the Fp157 genome and associated mass features detected in this study

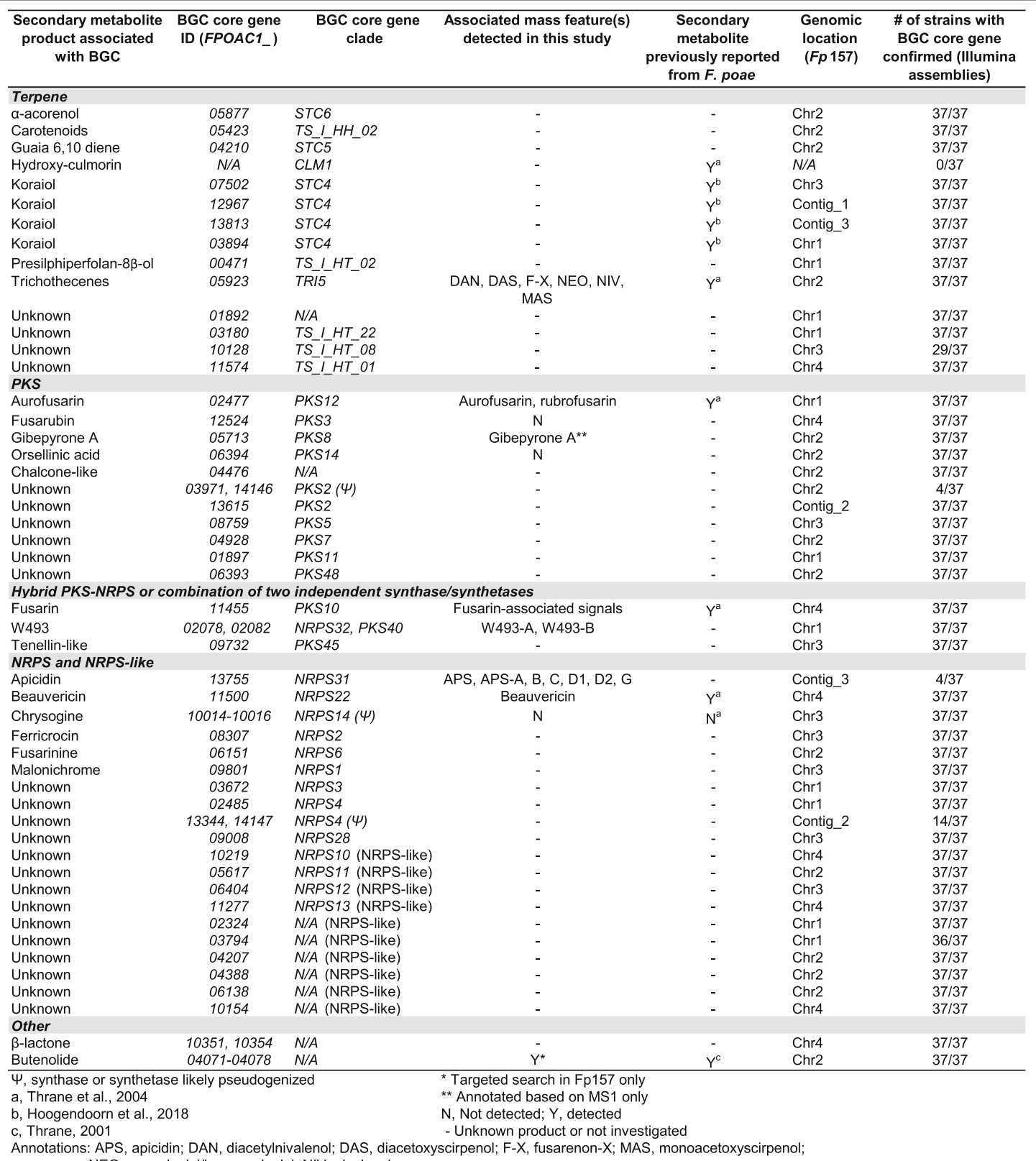
NEO, neosolaniol/isoneosolaniol; NIV, nivalenol

represents consensus chemical phenotypes from all isolates and includes all mass features discussed in this study as well as a subset of unannotated signals (see Additional file 7 for expanded analysis).

Hierarchical cluster analysis grouped the consensus chemical phenotypes ('metabolomes') by metabolite detection pattern similarities between isolates and between mass features (Fig. 2, left and top dendrograms). Mass features annotated as trichothecenes and fusarins were present in large clusters due to the many functional alterations present in these molecular families. Close examination of raw MS data from isolates Fp016 and
Fp038 revealed extracts from all media conditions were dominated by the relative abundance of fusarinassociated signals. This likely led to a skewed mass feature profile (with many mass features falling below the limit of detection) due to sample dilution prior to injection, and possibly again during data preprocessing, which could explain the absence of beauvericin and some trichothecene-associated signals from these isolates. Fusarins made up a significant portion of signals from nearly all isolates on all media types, with the exception of isolates $F p 059, F p 039$ and $F p 033$ which had lower frequencies of fusarin-associated signals relative to 


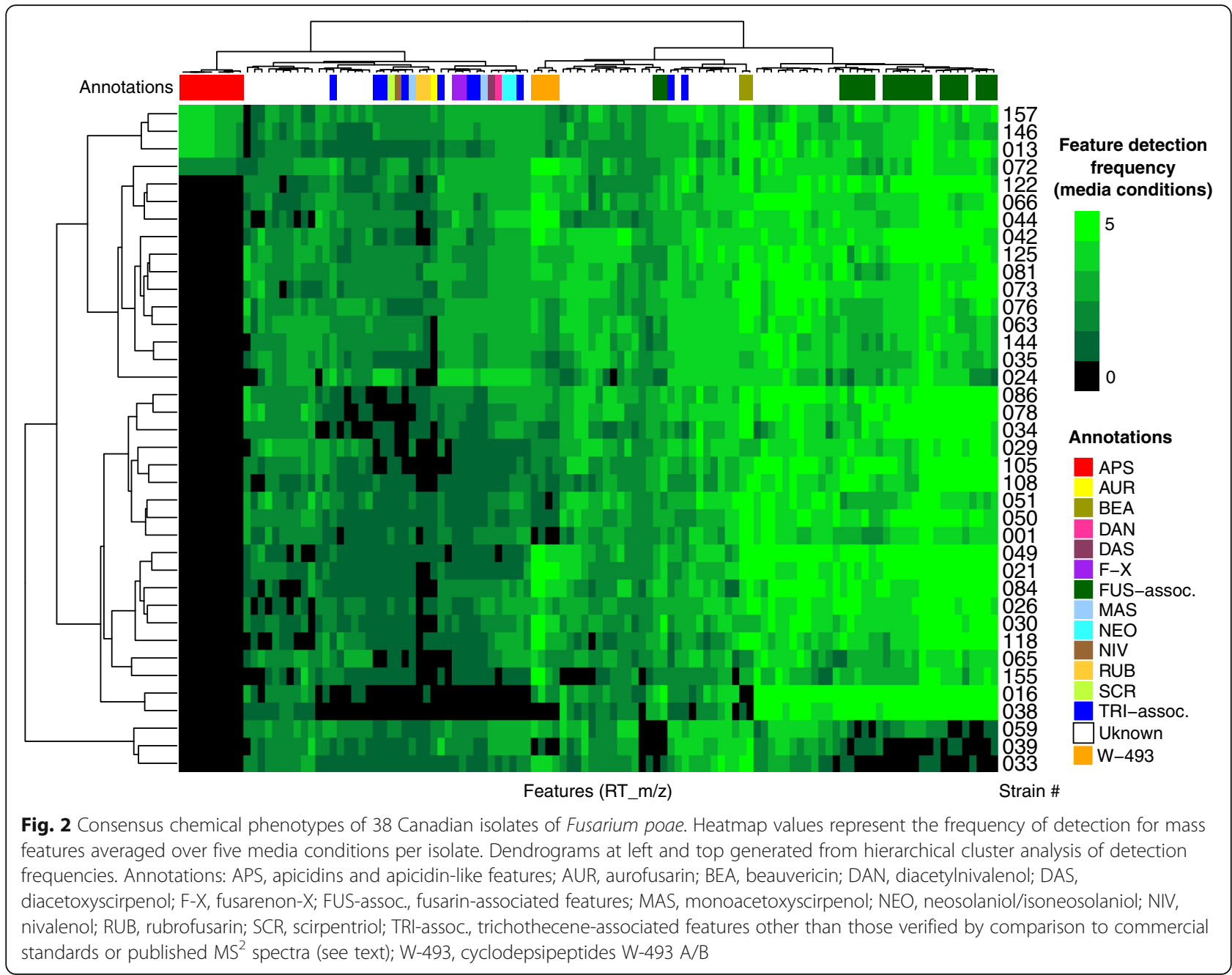

the other isolates. We concluded that isolates exhibited varying levels of core-chromosome associated signals. Furthermore, we noted four isolates, Fp157, Fp013, $F p 088$ and $F p 072$, exhibited mass features which were absent from all other isolate profiles, and were therefore predicted to be AC-associated based on the presumption that $\mathrm{AC}$ biosynthetic content varies across populations of $F$. poae. These mass features were annotated as apicidins.

\section{Annotation of type A and B trichothecenes}

Comparison of trichothecene-associated signals to commercial standards confirmed the presence of both types A and B which have been previously associated with $F$. poae. Isolates grown on YES media appeared to produce the most trichothecene-associated mass features, a pattern which is unsurprising since this media is rich in sucrose, a known trigger for trichothecene production in the closely-related species F. graminearum [36]. Mass features associated with 15-diacetoxyscirpenol, 15monoacetoxyscirpenol, neosolaniol and fusarenon-X were confirmed by comparison to standards. A feature with the same $\mathrm{m} / \mathrm{z}$ and similar fragmentation pattern as neosolaniol was detected, eluting slightly later than neosolaniol, and is therefore suggested to be iso-neosolaniol (either 4,8 diacetyl or 8,15 diacetyl form). Additionally, mass features were annotated as diacetylnivalenol and scirpentriol based on in silico fragmentation comparison. A mass feature matching a commercial nivalenol standard was detected from most isolates but was a lowintensity signal compared to other trichothecenes. Other signals associated with trichothecene biosynthesis matched $\mathrm{m} / \mathrm{z}$ and chemical formulas of trichothecene precursors such as isotrichotriol, or analogs of the major type A and B trichothecenes detailed above, and were tentatively annotated as "trichothecene-associated" due to the absence of publicly available $\mathrm{MS}^{2}$ spectra or commercial standards at this time.

3A-deoxynivalenol, 15A-deoxynivalenol, T-2, HT-2 and T-2 tetraol- associated $m / z$ were not detected from any isolate grown in this study. To test whether isolates had the genetic capability to produce T-2 or HT-2 toxin, 
we surveyed (by blastp) the $37 \mathrm{~F}$. poae Illumina genomes using the acetyl transferase TRI16 from F. sporotrichioides, shown to facilitate esterification of the C-8 hydroxyl group in trichothecenes during production of T-2 toxin [37], and no TRI16 homologs were detected.

\section{Confirmation of aurofusarin, beauvericin, fusarin, W-493 and other metabolites}

Next, we annotated mass features associated with nontrichothecene mycotoxins. Beauvericin was confirmed by comparison to a commercial standard. Cyclodepsipeptides W-493 A and W-493 B [38] were annotated by comparison to GNPS-supplied $\mathrm{MS}^{2}$ fragmentation spectra (Mirror plots comparing $\mathrm{MS}^{2}$ spectra are in Additional file 8). Fusarin-associated signals were numerous, likely owing to the multiple stereoisomers commonly observed from fusarin-producers [39, 40]. Features matching the $m / z$, predicted in silico fragmentation patterns and UV spectra of aurofusarin and its precursor rubrofusarin were detected from most isolates but production was not consistent to isolate groupings or media conditions. A mass feature matching $\mathrm{m} / \mathrm{z}$ of gibepyrone A was detected, but could not be confirmed due to lack of standards and experimentally derived $\mathrm{MS}^{2}$ spectral database representation, and in-silico $\mathrm{MS}^{2}$ spectra generation was inconclusive. Additionally, we searched for genomic and metabolomic evidence for production of butenolide, a cytotoxic secondary metabolite reported from F. poae [41]. A gene cluster homologous to the butenolide-associated cluster in F. graminearum [42] was detected in the $F p 157$ genome. We reasoned that due to the highly polar nature of butenolide, it likely eluted with the injection peak during chromatography and was therefore excluded from our UPLC-HRMS chemical phenotypes (the injection peak was sent to waste to prevent soiling of the MS inlet due to media components). Extracts from $F p 157$ were re-profiled by UPLC-HRMS without diverting the injection peak, and butenolide production was confirmed in this isolate (see Additional file 9 for details).

Several secondary metabolites predicted from the antiSMASH analysis for $F$. poae (Fp157) were not detected. Notable absences include fusarubin (and other analogs associated with the $f s r 1$ or $P K S 3$ cluster [43]), orsellinic acid and chrysogine. Fusarubin and orsellinic acid analogs may be among the unannotated signals here and will be the subject of further investigation. Closer inspection of the chrysogine-associated NRPS14 homolog in $F$. poae isolates indicates it has been disrupted by an $800 \mathrm{bp}$ insertion of very low GC content $(<10 \%)$ in all isolates including the Belgian $F$. poae isolate 2516. Although hydroxy-culmorins have been previously detected from $F$. poae isolates [9], we were unable to find homologs of the longiborneol synthase gene CLM1, shown to be required for culmorin production in F. graminearum [44], in any of the $F$. poae assemblies generated in this study. Hydroxy-culmorin-associated mass features were detected in the metabolomes of nearly all isolates (except $F p 016$ ), but lack support due to the absence of available commercial standards. The presence of hydroxy-culmorins is thus insufficiently supported at this time to warrant annotation.

Taken together, our data confirms Eastern Canadian $F$. poae isolates produce similar chemical profiles to European $F$. poae isolates when grown in vitro, as pertaining to trichothecene, fusarin, beauvericin and aurofusarin production. Our untargeted analysis of chemical profiles detected cyclodepsipeptides W-493-A and B associated signals, and highlighted mass features not matched in our database of known Fusarium mycotoxins which could represent undescribed secondary metabolites (Fig. 2).

\section{The production of apicidin and its derivatives is linked to the accessory chromosome in Fp157}

We investigated the detection of apicidins. Mass features were detected primarily from broth extracts of four of the 38 isolates cultured ( $F p 013, F p 072, F p 146, F p 157)$, and included features with $\mathrm{m} / z$ and isotope ratios matching apicidin (APS) and APS analogs A, B, C, D1, $\mathrm{D} 2$, and $\mathrm{G}[45,46]$. To confirm these annotations, we used feature-based molecular network analysis and $\mathrm{MS}^{2}$ spectral matching (Fig. 3a). Network analysis showed all isolate-specific signals grouped into a single subnetwork. $\mathrm{MS}^{2}$ spectra from three mass features matched publicly available, experimentally-derived $\mathrm{MS}^{2}$ spectra from APS, APS B and APS C (mirror plots in Additional file 10). $\mathrm{MS}^{2}$ data from APS-A, D1, D2 and G, were not represented in experimentally-derived $\mathrm{MS}^{2}$ databases at the time of analysis. However, APS-A, D1 and D2 were supported by in silico predictions of potential fragmentation patterns from known molecular structures using Sirius / CSI Finger-ID. Lastly, APS G was annotated by manual examination of the $\mathrm{MS}^{2}$ spectra (Fig. $3 \mathrm{~b}$, see Additional file 11 for expanded analysis of APS G signals, and Additional file 12 for expanded network analysis of APSassociated signals). Molecular networking analysis indicated there were at least three unannotated signals which matched fragmentation patterns of the apicidinlike signals, suggesting they are novel APS analogs. The most intense of the three unknown APS-like signals had the same $m / z$ as apicidin D1 $[\mathrm{M}+\mathrm{H}]^{+}$peak $(m / z$ $640.3705)$ but was determined to be a $\left[\mathrm{M}-\mathrm{H}_{2} \mathrm{O}+\mathrm{H}\right]^{+}$ion by the MZMine IIN module, with the $[\mathrm{M}+\mathrm{H}]^{+}$missing from the raw data, although a $[\mathrm{M}+\mathrm{Na}]^{+}$adduct signal was evident. The other two were detected at relatively low intensities and were not further investigated. 


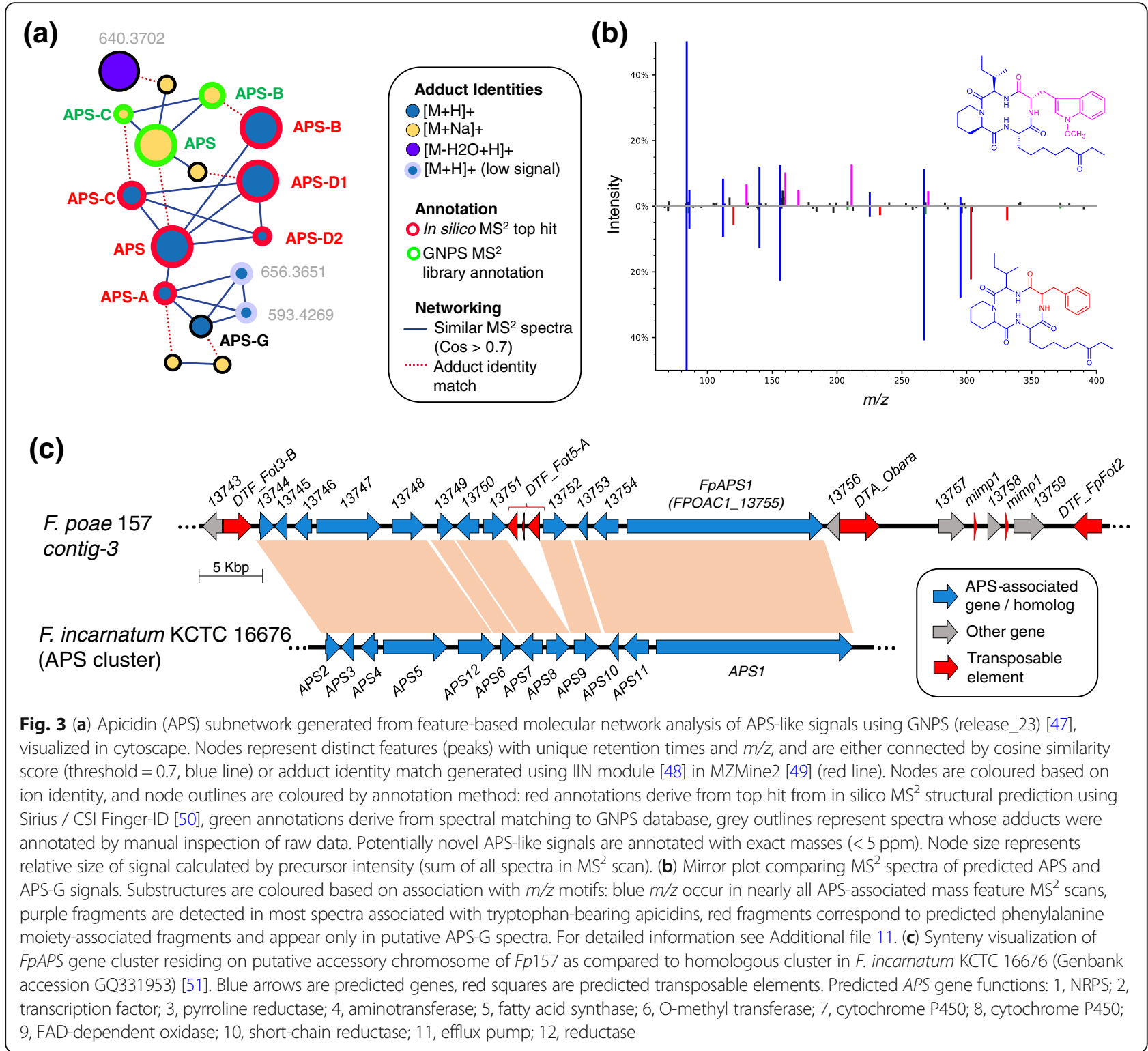

Genomic analysis of the $F p 157$ isolate revealed homologs of all 11 genes previously shown to be co-regulated during APS production [51], are present in a single, near-contiguous cluster located on AC-associated contig_3. We annotated this cluster as 'FpAPS'. We compared the FpAPS cluster to the APS cluster annotated from Jin et al. 2010 using blastn (Fig. 3c) and determined all genes relating to APS production are found in the same relative order, with the exception of a DNAtransposon insertion between APS8 and APS9 in Fp157. This transposon matches DTF_Fot5-A from a previously published library of transposons described from the Belgian F. poae isolate 2516 [17], but appears to be fragmented (Fig. 3c).

Additionally, four genes with predicted roles in biosynthesis were found adjacent to the FpAPS cluster in
Fp157 and are uniquely present in APS-producing isolate genomes. The co-localization of these genes to the FpAPS cluster indicates they may be involved in APSassociated molecular family diversification. FPOAC1_ 13756 is a predicted amidase, which appears to have a DTA_Obara DNA transposon insertion (see [52] for explanation of TE nomenclature used here). This gene is also present in the homologous APS cluster in F. sporotrichioides and has not previously been associated with apicidin production. Three additional predicted biosynthetic genes are adjacent to the FpAPS cluster: FPOAC1_13757 is an NRPS-like gene with an AMPbinding domain, FPOAC1_13758 is an O-methyl transferase and FPOAC1_13759 is an oxidoreductase with similarity to the ELFV-dehydratase family. Interestingly, when searched against the NCBI database, all three 
genes have top blastn hits in a three-gene cluster encoded by the strawberry anthracnose-causing mold, Colletotrichum nymphaeae isolate SA01, which is suggested to grow endophytically in weedy grasses [53]. Furthermore, the trio of genes are interspersed by two repetitive sequences with homology to 'miniature-impala' or 'mimp' TEs which have been associated with pathogenicity genes on Fusarium oxysporum ACs [54]. The FpAPS cluster and adjacent genes are present in the genomes of all isolates from which APS-associated mass features were detected.

The possibility of the FpAPS cluster originating by horizontal gene transfer from another species was investigated by performing a Blastn analysis of the FpAPS genes against the NCBI nucleotide database. Top hits for all $F p$ APS genes matched coding sequences from close relatives $F$. sporotrichioides and F. langsethiae, with most at $\sim 97 \%$ nucleotide identity, are provided in Additional file 13. As assemblies for F. sporotrichioides and F. langsethiae are not currently at chromosome-level, we cannot yet infer whether their APS-like BGCs are on ACs or core chromosomes. However, the location of the contig breaks in the F. langsethiae 201059 genome strongly suggests that it has the same transposable element (TE) insertion site between APS 8 and APS9 and therefore this APS-like cluster is likely to be either on an AC or accessory region in this isolate.

Finally, to assess the frequency of occurrence of FpAPS1 in our collection of 193 Ontario and Quebec F. poae isolates as well as 10 international isolates, we designed PCR primers which would specifically amplify a $150 \mathrm{bp}$ nucleotide sequence from $F$. poae APS1 and screened the genomic DNA (see Additional file 14 for representative sample gel lane). Of these, 15 isolates tested positive for the APS1 gene, including an isolate collected from Ontario wheat in 2006 (DAOMC 239526), indicating the presence of the FpAPS1 has persisted at least as long as the time period under study in Eastern Canada. See Additional file 1 for results of APS1 screening. Active APS production was confirmed from 4 out of the 15 FpAPS1 containing F. poae isolates (as they were the only ones included in the in-depth metabolomics analysis described above). None of the international isolates tested positive for the APS1 gene.

\section{Discussion}

In this study we have examined the secondary metabolite biosynthetic potential of 38 isolates of $F$. poae from Eastern Canada by analysis of genomes and chemical phenotypes. The combination of modern genome sequencing platforms and UPLC-HRMS profiling of fungal extracts provides a powerful approach for screening communities of fungal plant pathogens which may exhibit lineage-specific metabolite traits.
In this case, untargeted chemical profiling enabled the confirmation of known mycotoxins associated with a 'core' F. poae chemical phenotype in addition to the discovery of an 'accessory'-associated metabolome present only in a subset of isolates. These isolates are producing known and potentially novel forms of APS, a potent histone deacetylase inhibitor [55]. The highquality genome of an APS-producing isolate, Fp157, indicates there are many biosynthetic gene clusters in this species which have not yet been associated with known products. Moreover, the presence of secondary metabolite BGCs on ACs can further diversify chemical phenotypes, underlining the desirability of untargeted metabolomic screening of population isolates to detect novel mycotoxin signatures.

Core chromosome-associated secondary metabolites described in this study generally agree with previously published data from European $F$. poae isolates cultured in vitro [56], with some minor exceptions. Production of the highly toxic T-2 and HT-2 toxins in grains infected with $F$. poae has been described $[9,57]$ but is not supported by recent genetic and chemotype analyses [56] including this study. The absence of TRI16 in F. poae genomes generated here indicates Eastern Canadian isolates are unlikely to produce $\mathrm{T}-2$ or $\mathrm{HT}-2$ toxins regardless of the experimental conditions employed. Although it is possible that a TRI16 homolog could reside on a isolate-specific AC or accessory region in other isolates, we believe it is also likely that previously reported F. poae T-2 and HT-2 producers were misidentified isolates of $F$. langsethiae, a known producer of T-2 and HT-2 with a very similar morphology to $F$. poae.

In addition to known mycotoxin confirmation, this study highlights undescribed biosynthetic potential in $F$. poae populations. From a genomics perspective, this includes roughly half the PKS clusters detected in Fp157, which have no predicted products (Table 1). Among these, PKS clades 7 and 8 are considered to be ubiquitous among all studied Fusaria, clade 5 is discontinuously distributed within Fusarium, and clades 45 and 48 are present in only a few Fusaria [35]. Similarly, products of some NRPS and NRPS-like clusters in $F$. poae are undescribed, including NRPS clades 3, 4 and 10-13 (all appear common among Fusaria [15]). Preliminary analysis suggests some of the unannotated metabolomics signals presented here may represent products of undescribed BGCs and provide targets for further molecular elucidation.

APS production and APS-associated BGCs have been detected from numerous Fusarium species at various levels of phylogenetic distance from $F$. poae, however the origins of this cluster in $F$. poae isolates remain unclear. APS was first detected from an isolate of the Fusarium incarnatum-equiseti species complex (FIESC) 
[58] and the gene cluster has since then been detected from Fusarium isolates across six species complexes [14, 59] including FIESC-12 (isolate NRRL 66336), which has since been reclassified as Fusarium flagelliforme [60], and FIESC-26 (isolate ATCC 74289 [58]), reclassified as Fusarium hainanense [60]. In vitro production of apicidins is confirmed from the FIESC [45, 58, 61], F. langsethiae [62], F. fujikuroi [63], and possibly $F$. sambucinum (isolates KCTC 16676 and 16677, identified by morphology only). The presence of APS-like clusters among diverse Fusaria suggests horizontal transfers of genes or ACs could be at play. However, blastn comparisons indicate the FpAPS genes share highest nucleotide identities to the closest known relatives of $F$. poae, including $F$. sporotrichioides and $F$. langsethiae (Additional file 13). This makes it challenging to predict whether the presence of FpAPS in Fp157 is the result of horizontal transfer from a close relative, or whether the cluster originates from a common ancestor and is retained by a small number of $F$. poae isolates. It is beyond the scope of this study to resolve this problem. More high quality long-read genomes will help unravel the evolutionary path of this BGC in Fusarium.

Although apicidins have demonstrated phytotoxicity towards wheat and maize [64], and were expressed by pathogenic $F$. fujikuroi isolates during growth in rice [65], their role during infection by plant pathogens, if any, is unknown. Apicidins have traditionally been recognized for their potent ability to inhibit histone deacetylase activity in apicomplexan parasites [55], and their use as antitumor therapeutics [66, 67]. Histone deacetylase inhibition can lead to hyperacetylation of histones, impacting an organism's ability to regulate genetic transcription. Apicidins are structurally similar to HC-toxin, another cyclic tetrapeptide histone deacetylase inhibitor with a well-documented role as a virulence factor during infection by the fungal plant pathogen Cochliobolus carbonum [68]. The recent detection of $\mathrm{HC}$-toxin gene clusters in the genomes of Alternaria brassicae isolate J3 [69] (where it is assembled to a putative AC) and Alternaria jesenskae isolate AM237084 [70] suggests this cluster may have been horizontally transferred between species or even genera. As with the FpAPS cluster, the evolutionary origin of the HC-toxin cluster in Alternaria has not been ascertained. A recent study comparing chromosome counts found evidence for ACs in many FHB-associated APS producers, including F. avenaceum, F. poae, F. sporotrichioides, and members of the F. incarnatum-equiseti species complex [71]. Long-read genome sequencing of these Fusaria will cast light on whether the APS cluster is on an AC or accessory region in these species.
FpAPS was not the only secondary metabolite BGC identified on AC-associated contigs in Fp157. Paralogous genes from clades NRPS4, PKS2 and STC4 were represented on both core chromosome and AC-associated sequences, with paralogs sharing 70-80\% nt identity (Table 1, Fig. 1). Although TE-disruption has likely pseudogenized the core chromosome-associated PKS2 and AC-associated NRPS4 paralogs in Fp157, their presence support the possibility of historical gene amplification and/or neofunctionalization (Fig. 4). It is unclear whether FpPKS2 and/or the disrupted FpNRPS4 located on predicted ACs originate from the duplication of core chromosome genes, interspecies hybridization (followed by chromosome/gene losses), or horizontal AC transfer from another species. By contrast, genomic evidence from $F p 157$ and Belgian $F$. poae isolate 2516 suggests gene duplication has occurred for STC4 paralogs on ACs; three copies with greater than $98 \%$ nt ID were assembled in $F p 157$ and over six copies were assembled in F. poae 2516. Furthermore, the localization of one of the STC4 copies to a subtelomeric region of a core chromosome in $F p 157$ underlines the potential for interchromosomal gene transfer of biosynthetic genes between ACs and core chromosomes in F. poae. Koraiol is the predicted product of STC4 paralogs in F. poae 2516, and has been recently associated with pathogenic $F$. fujikuroi isolate growth in planta [65]. Clarifying the effects of koraiol synthase in planta will help generate testable hypotheses on the effects of its multiplication in $F$. poae genomes.

Mapping the various secondary metabolite clusters associated with ACs onto the $F$. poae BUSCO phylogeny presents a dynamic picture of $\mathrm{AC}$-associated genes in $F$. poae. As seen in Fig. 5, the BUSCO-inferred clades divide into two groups based on widespread presence of FpNRPS4 ( $\Psi)$ paralog variants. In one group, isolates have complete FpNRPS4 ( $\Psi)$ representation (100\% coverage when compared to $F p 157)$, although in every instance FpNRPS4 ( $\Psi$ ) is split between contigs, at the same site as the TE disruption in Fp157, implying the synthetase has been disrupted in all genomes in which it appears. The second group contains a truncated FpNRPS4 ( $\Psi$ ) fragment with $15 \%$ coverage, suggesting the synthetase has further degraded in this lineage. Mating type MAT1-1 is the dominant mating type in this population, as was found in European populations [56]. Potential markers for ACs, including FpPKS2, FpNRPS4 $(\Psi)$ fragments, STC4 paralogs, and Zit1 (a small TE previously associated with ACs in $F$. poae [72], data not shown), are detected in nearly all genomes, supporting the possibility of widespread ACs in F. poae populations.

The effects of $\mathrm{AC}$-associated genes on $F$. poae isolate pathogenicity, if any, remain unexplored. Extensive profiling of $F$. poae populations is currently being 


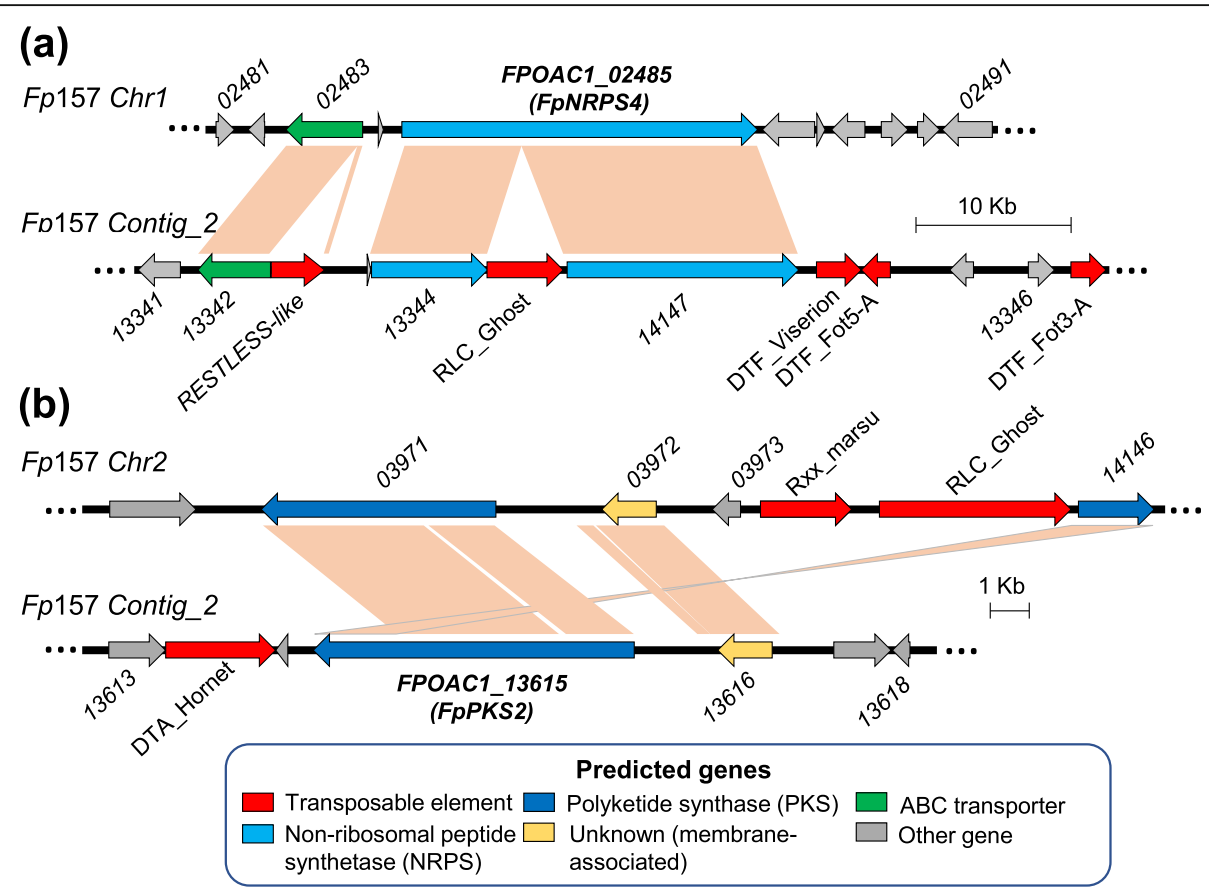

Fig. 4 Gene synteny visualization of predicted BGCs on Fp157 core chromosomes and homologous regions on accessory chromosomes. (a) FPNRPS4-associated region on core chromosome 1 compared to homologous region on Contig_2. (b) FpPKS2-associated region on core chromosome 2 compared to homologous region on Contig_2

undertaken in a Canada-wide survey to build on the work presented here, in combination with in planta pathogenicity trials to further explore the role of $F$. poae in the FHB disease complex. Critical issues remain outstanding with regards to $F$. poae, which would help shape hypotheses surrounding the utility of its secondary metabolite outputs: what is the complete life cycle of this fungus? What are its primary hosts or trophic states (pathogenic, saprotrophic or endophytic)? Are some isolates expanding their distribution at the expense of others?

Fusarium poae has been flagged as a potential danger to agriculture, and for good reason: the mycotoxins and emerging mycotoxins detected from this species have demonstrably harmful effects to living cells, disrupting key processes such as protein synthesis (trichothecenes), DNA transcriptional control (APS) and compartmentalization of ion gradients (beauvericin) [73]. Apicidin is not currently monitored in Canadian cereals and is not regulated anywhere globally. In a recent study of mycotoxin content in globally-sourced pig feeds, APS was detected in over half the feed samples and was found to be the most cytotoxic against pig gut endothelial cells in comparison with 27 other mycotoxins detected [74]. Less is known of the effects APSs might have on plant systems and the evolution of pathogenicity. For example, although the bioactivity of APS is well documented and likely not trivial to the plant infection process, there is as yet insufficient evidence to support the hypothesis that the presence of the FpAPSbearing $\mathrm{AC}$ or any other $\mathrm{AC}$ associated with the isolates studied here improves the fitness of $F$. poae in the context of cereal crop invasion. Nevertheless, the potential ability of FHB-associated Fusaria to transfer and modify BGCs between species via ACs and other rapidlyevolving genetic compartments is surely worrisome for plant breeders. This justifies a careful examination of the genomic and biosynthetic potential of FHB-associated Fusaria, which will help us to understand how they may evolve to invade new niches and overcome plant defenses. Given their highly toxic nature and the frequency of predicted production among FHB-associated Fusaria, we believe further studies of APS and APS-producing fungi are warranted - particularly among oat pathogens.

\section{Materials and methods}

\section{Source of isolates}

Fusarium poae isolates selected for study were obtained with permission from the research culture collection of Dr. Allen Xue [4] maintained at Agriculture \& AgriFood Canada (Ottawa, ON) (See Additional file 1 for complete list). A subset of these isolates for which genome sequences were deposited in the Canadian Collection of Fungal Cultures (CCFC, Agriculture \& Agri-Food Canada, Ottawa, ON). Historic isolates were also sourced from the CCFC (10 Canadian and 10 


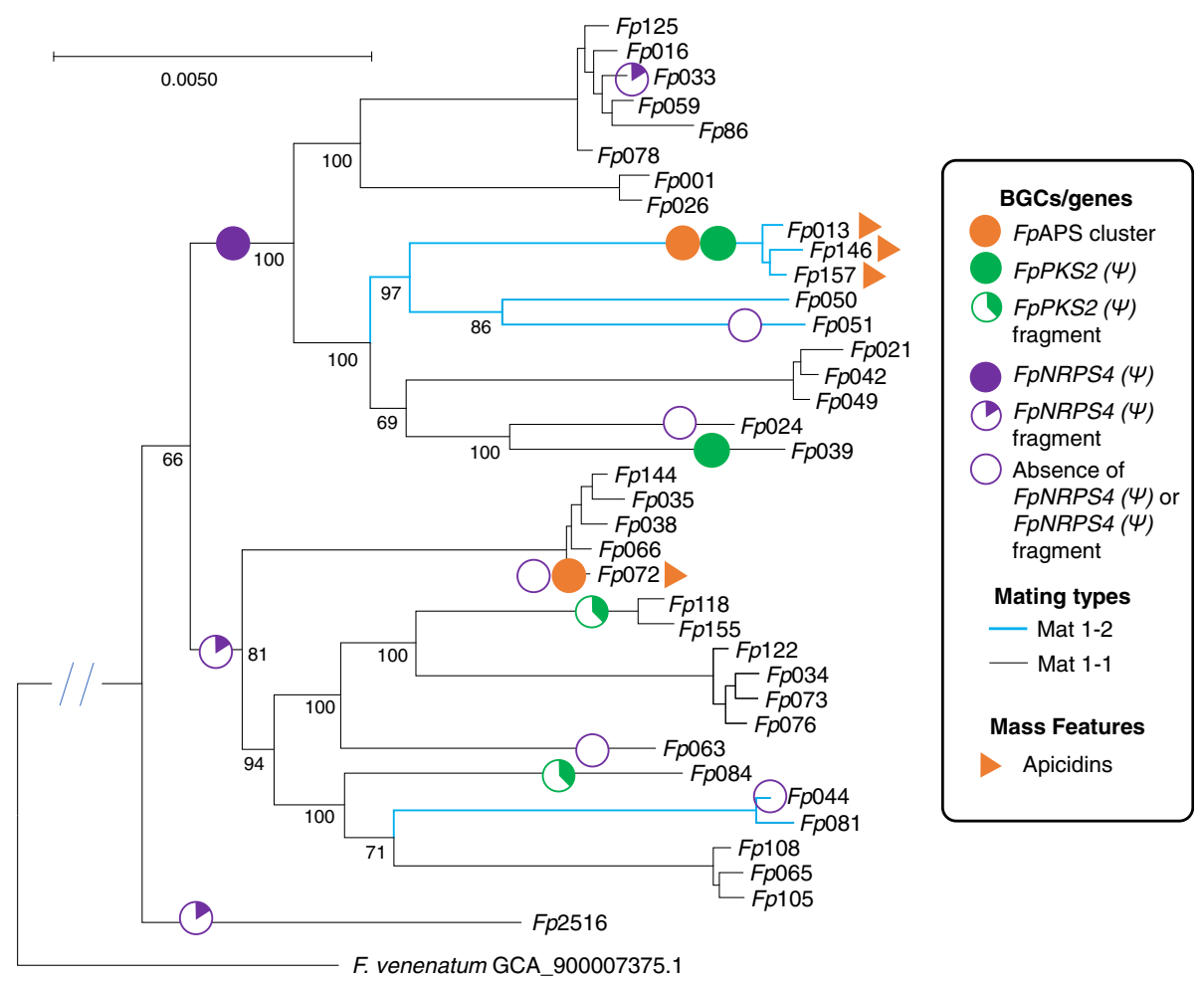

Fig. 5 Phylogenetic analysis of 4141 single-copy BUSCO orthologs from F. poae isolates investigated in this study including Belgian isolate 2516. F. venenatum assembly GCA_900,007,375.1 was included as an outgroup to determine the true location of root (double slashes indicate truncated branch leading to outgroup). Evolutionary histories were inferred using the Maximum Likelihood (ML) method. Branch lengths are measured in number of substitutions per site, and node values indicate Ultrafast bootstrapping values $(n=1000)$. Biosynthetic gene annotations: the FpAPS cluster and the disrupted FPNRPS4 ( $\Psi$ ) synthetase are associated with accessory chromosome sequences in the Fp157 assembly, whereas the disrupted FPPKS2 ( $\Psi)$ synthase was assembled to a core chromosome in Fp157. Purple and green pie charts represent size of fragments detected relative to concatenated Fp157 FpNRPS4 $(\Psi)$ or PKS2 $(\Psi)$ sequences. Empty pie charts indicate absence of FpNRPS4 $(\Psi)$ detection

international). A preliminary assessment of genetic and potential mycotoxin diversity was made by amplification of TEF1- $\alpha$ and TRI genes (TRI1 \& TRI8) (Additional file 2).

\section{DNA extractions, PCR and Sanger sequence analysis}

Single-spore cultures were grown on half-concentration potato dextrose agar (PDA, BD Difco Brand, NJ, USA) plates at $25^{\circ} \mathrm{C}$ until nearly confluent. Plugs were transferred to a PDA plate and grown at $25^{\circ} \mathrm{C}$ until confluent for genomic DNA extraction and on a synthetic nutrient agar (SNA) plate for 8 days at $25^{\circ} \mathrm{C}$ with UV light to prepare frozen glycerol spore stocks.

Fresh mycelium was collected from the PDA plate and placed in a $2 \mathrm{~mL}$ screw-cap tube with one $1 / 4$ " Ceramic Sphere and Lysing Matrix (M.P. Biomedicals). DNA was isolated using the E.Z.N.A. Fungal DNA Mini Kit (Omega Bio-tek Inc.), lysing the tissue in FG1 buffer using a FastPrep24 Sample Preparation System (M.P. Biomedicals). Genomic DNA was eluted in $50 \mu \mathrm{L}$ Elution Buffer.

F. poae-specific primers for TEF1 $\alpha$, TRI1 and TRI8 were designed based on the published $F$. poae genome
$[17,24]$ (Additional file 15). PCR amplification was performed with the Advantage 2 PCR Polymerase Mix (Takara Bio USA, Inc.) with $200 \mathrm{nM}$ of each primer in a $25 \mu \mathrm{L}$ reaction volume, and three-step amplification with annealing at $59^{\circ} \mathrm{C}$ for 30 cycles. Following amplification, $3 \mu \mathrm{L}$ was run on a $1 \%$ agarose gel. The PCR product was purified using the GenepHlow Gel/PCR kit (Geneaid Biotech Ltd.) as per manufacturer's instructions and DNA eluted in $30 \mu \mathrm{L}$ Elution buffer. A $20 \mathrm{ng}$ aliquot of each purified PCR product was sequenced with the forward and reverse primer using a BigDye Terminator v3.1 Cycle Sequencing Kit (Applied Biosystems) as described by the manufacturer in a $10 \mu \mathrm{L}$ reaction volume with primer concentration of $3.2 \mu \mathrm{M}$. After precipitation, reactions were run on an ABI 3500xl Genetic Analyzer (Applied Biosystems). Sequence analysis and alignment was done using the Lasergene12 Core Suite (DNASTAR Inc.) and Geneious R11 (Biomatters Ltd.).

Fermentation, solvent extraction and UPLC-HRMS analysis Frozen isolate stocks were thawed and inoculated into slants containing $15 \mathrm{~mL}$ of liquid media, where they grew at $25^{\circ} \mathrm{C}$ in the dark (3 replicates per isolate per 
medium). Media conditions included MMK2, CYA, YES, YES with salt-added ("YESIO"), and S2M broth (Additional file 6). After 14 days of incubation, mycelial mats were removed from the liquid media for all conditions except for S2M. For the S2M treatment, isolates were grown at $28^{\circ} \mathrm{C}$ in $\mathrm{S} 1 \mathrm{M}$ for 4 days, and then transferred to slants containing S2M for 6 days of incubation. In all media conditions, after centrifugation of the supernatants to remove residual mycelia, broth and mycelia were extracted separately in $125 \mathrm{~mL}$ Erlenmeyer glass flasks with $15 \mathrm{~mL}$ of ethyl acetate for $1 \mathrm{~h}$, shaking at $120 \mathrm{rpm}$ at room temperature. Solvent supernatants were transferred to pre-weighed borosilicate scintillation vials and dried under vaccuum. Extracts were reconstituted in methanol to a concentration of $500 \mu \mathrm{g} / \mathrm{mL}$ and analyzed on a Thermo Ultimate 3000 UPLC coupled to a Thermo LTQ Orbitrap XL high resolution mass spectrometer and a Thermo Dionex Ultimate 3000 Diode array detector $(190-800 \mathrm{~nm})$. Chromatography was performed on a Phenomenex $\mathrm{C}_{18}$ Kinetex column $(50 \mathrm{~mm} \times 2.1 \mathrm{~mm}$ ID, $1.7 \mu \mathrm{m}$ ) with a flow rate of $0.35 \mathrm{~mL} / \mathrm{min}$, running a gradient of water $(+0.1 \%$ formic acid $)$ and acetonitrile $(+0.1 \%$ formic acid): starting at $5 \%$ acetonitrile increasing to $95 \%$ acetonitrile by 4.5 mins, held at $95 \%$ acetonitrile until 8.0 mins, returning to $5 \%$ acetonitrile by 9 mins and held to 10 mins to equilibrate the column to starting conditions. The HRMS was operated in $\mathrm{ESI}^{+}$ mode (monitoring a range of $100-2000 \mathrm{~m} / \mathrm{z}$ ) using the following parameters: sheath gas [40], auxiliary gas [5], sweep gas [2], spray voltage $(4.2 \mathrm{kV})$, capillary temperature $\left(320^{\circ} \mathrm{C}\right)$, capillary voltage $(35 \mathrm{~V})$, and tube lens $(100 \mathrm{~V})$.

$\mathrm{MS}^{\mathrm{n}}$ fragmentation was performed in high resolution on select ions in subsequent experiments using CID at $35 \mathrm{eV}$. MassWorks ${ }^{\mathrm{Tm}}$ software (v5.0.0, Cerno Bioscience) was used to improve spectral accuracy and confirm the molecular formulas of annotated ions. The sCLIPS searches were performed in dynamic analysis mode with elements $\mathrm{C}, \mathrm{H}, \mathrm{N}$, and $\mathrm{O}$ allowances set at minimum 1 and maximum 100. Charge was specified as 1 and mass tolerance set to $5 \mathrm{ppm}$.

Additional experiments were performed to investigate the diversity of apicidin-like signals in isolate extracts via generation of $\mathrm{MS}^{2}$ data. This work was performed using a Thermo Q-Exactive Plus mass spectrometer (Thermo Fisher Scientific). See Additional file 16 for details.

\section{Metabolomics processing and visualization}

A detailed explanation of parameters used for metabolomics data processing are provided in Additional file 16. In brief, data preprocessing was carried out using a version of MZMine v2.37 which includes the Ion Identity Networking module [49]. Raw data files were converted into a data matrix of discriminate variables, each being a combination of retention time (RT) and $m / z$. Variables are designated here as mass features, with intensities calculated based on peak area measurements. Data was imported into the $\mathrm{R}$ environment, where mass features representing associated adducts and in-source fragments of the same parent ion were grouped using Pearson correlation analysis over a sliding window of elution time. These groupings were compared to data generated during raw data preprocessing via the Ion Identity Networking module which correlated peak shapes of coeluting signals [48]. Mass feature data from the two extraction types (mycelium and broth) were summed, converted to binary form, and then averaged across the five media conditions to form a 'pseudo-binary' matrix of detection frequencies for each mass feature.

\section{Mass feature annotation}

Wherever possible, mass features were annotated by comparison of exact $m / z(<5 \mathrm{ppm})$, retention time and $\mathrm{MS}^{2}$ fragmentation pattern to commercial standards. 3Adeoxynivalenol, enniatin-A, enniatin- $A 1$, enniatin-B, enniatin-B1 were purchased from Sigma Aldrich (St. Louis, USA). Beauvericin and trichothecene standards 3, 15-diacetoxyscirpenol, 15-monoacetoxyscirpenol, neosolaniol, T-2, HT-2, T-2 tetraol, fusarenon-X and nivalenol were purchased from Fermentek (Israel). A 15Adeoxynivalenol standard was purified in-house. Where standards were not available, chemical formulas were predicted based on high resolution exact masses of $[\mathrm{M}+\mathrm{H}]^{+}$ or $[\mathrm{M}+\mathrm{Na}]^{+}$ions $(<5 \mathrm{ppm})$ combined with isotope abundance patterns (analyzed using MassWorks, Cerno Bioscience). Annotations were supported by analysis of $\mathrm{MS}^{2}$ fragmentation patterns using SIRIUS / CSI Finger-ID [50] for in silico predictions and also using the MASST search tool as part of the GNPS workflow $[47,75]$ for comparison to experimentally derived $\mathrm{MS}^{2}$ spectral databases. Additionally, annotations were supported wherever possible by comparison of UV absorbance spectral signatures. To further support the annotation of apicidins, we generated $\mathrm{MS}^{2}$ scans of all related signals using a Thermo QExactive mass spectrometer, and performed feature-based molecular network analysis using MZMine2 [49] (special pre-release version 2.37.1corr17.7) and GNPS. Structural hypothesis generation was assisted by the use of mass motif finding using MS2LDA [76].

\section{Genomic DNA isolation, genome sequencing, assembly and annotation}

To generate genomic DNA (gDNA) for both Illumina and Nanopore genome sequencing, spores from F. poae isolates (see Additional file 3 for list of isolates) were inoculated in $250 \mathrm{~mL}$ Erlenmeyer flasks containing $50 \mathrm{~mL}$ first-stage media (Miller and Blackwell, 1986) and incubated at $26^{\circ} \mathrm{C}$, shaking at $170 \mathrm{rpm}$, for 4 to 5 days. 
Filtered fungal mycelia were flash frozen and ground in liquid nitrogen with a mortar and pestle until a fine powder was produced. Genomic DNA was extracted using the Illustra Nucleon PhytoPure DNA Extraction Kit (GE Healthcare Bio-Sciences), as per manufacturer's instructions. The gDNA pellet was reconstituted in $200 \mu \mathrm{L} 10 \mathrm{mM}$ Tris $\mathrm{pH} 8.0$ and the gDNA concentration determined using a FLUOstar OPTIMA fluorometer (BMG LABTECH) and a PicoGreen dsDNA Quantitation Kit (Molecular Probes Inc.). The reconstituted gDNA was mechanically sheared to $\sim 300$ bp fragments with a Covaris LE220 instrument and used as a template to construct PCR free Libraries with NxSeq AmpFREE Low DNA Library kit (Lucigen) and TruSeq CD dual indices (Illumina) according to the Lucigen's Library protocol. Indexed libraries were pooled, and sequencing was carried on a NextSeq500/550 (Illumina) using $2 \times$ 150 bp NextSeq High Output Reagent Kit (Illumina) according to the manufacturer's recommendations in order to obtain paired-end reads. Genome assembly using the generated Illumina data was performed with SPAdes v3.10.1 [77].

For Nanopore long-read gDNA sequencing of $F p 157$, gDNA was isolated from $500 \mathrm{mg}$ of frozen tissue using the Illustra Phytopure Genomic DNA Extraction kit (GE Healthcare). Approximately $6 \mu \mathrm{g}$ gDNA was then fractionated with a $0.75 \%$ agarose gel cassette $(10 \mathrm{~kb}-40 \mathrm{~kb})$ using a SageELF instrument (Sage Science Inc., USA) and the three most abundant fractions were combined for Nanopore sequencing selecting for long reads (SQKLSK109) using the revC protocol (Oxford Nanopore Technologies, UK) with the following modifications. To reduce loss of DNA after each elution, the initial $70 \mu \mathrm{L}$ of AMPure XP beads (Beckman Coulter Life Sciences, USA) were reused throughout the procedure. Instead of being transferred to a new tube, the eluted DNA remained in the tube with the AMPure XP beads. Where the addition of new AMPure XP beads was indicated in the protocol, a filter-sterilized 20\% PEG $8000 / 2.5 \mathrm{M}$ solution was added instead. Incubation times throughout the protocol were increased to $10 \mathrm{~min}$. The Long Fragment Buffer (LFB) was used in the adapter ligation step. The library yield was $53 \mathrm{ng}$. The DNA library was loaded on a FLO-MIN106 flow cell as described in the protocol and run with a MinION device (Oxford Nanopore Technologies, UK) for $48 \mathrm{~h}$.

Genome assembly of $F p 157$ was performed with CANU v1.8 [78] using the sequenced Nanopore reads with default settings with an estimated genome size of $40 \mathrm{Mb}$ (genomeSize $=40 \mathrm{~m}$ ). The first correction of the CANU assembly was performed using Nanopolish v. 0.11.1 [79]. Illumina reads of $F p 157$ generated from genomic DNA were mapped to the Nanopolish-corrected CANU assembly with BWA v0.7.17 [80] and additional errors were corrected with Pilon v1.23 [81]. Using Geneious, contigs were aligned and compared with those of F. poae 2516 (LYXU01) [17] to identify chromosomes $1-4$, the mitochondrial contig, and putative ACs of Fp157.

To prepare for genome annotation, transcriptome assemblies of $F$. poae 2516 [17] and another isolate, $F p 133$, grown in MMK2 and YES (data not shown), were performed with Trinity v2.8.5 [82] with default settings, from the Illumina sequenced RNA. FUNANNOTATE v1.5.2 [83] was used to annotate the $F p 157$ polished genome using the standard protocol. Gene prediction was performed with the assembled transcriptomes of $F$. poae 2516 and $F p 133$ used as transcript evidence. Two predicted genes were manually added to the assembly: FPOAC1_14145 and FPOAC1_14146 were annotated based on blastn match to FpPKS2 and FpNRPS4 (see Fig. 4).

Following annotation, the command-line version of antiSMASH 5.1 was used to predict the location of biosynthetic gene clusters [34]. Repetitive elements were detected using RepeatMasker and annotated using a merged database consisting of the 2018 version of RepBase [84] and previously annotated repetitive elements from $F$. poae isolate 2516 [56].

PKS and NRPS clade nomenclature used in this publication refers to the published clades from which either the NRPS adenylation domains or the PKS coding regions score highest in blastx comparisons (for example, the PKS12 cluster in $F$. poae contains a PKS with homology to those in clade 12, which has been associated with an aurofusarin production). Because Fusarium terpene synthase nomenclature has not yet been standardized, we adopted terpene synthase clade names associated with studies of $F$. langsethiae and F. fujikuroi where applicable $[62,65]$.

\section{BUSCO analysis}

A total of 4141 single-copy orthologues of housekeeping genes associated with Hypocreales (database: Hypocreales_odb10) were identified from Illumina assemblies from nearly all isolates in this study ( $F p 030$ was removed due to poor genome sequence quality) including Belgian $F$. poae isolate 2516, using BUSCO v4.0.5 [28]. Nucleotide sequences were aligned using MAFFT v7.470 [85] and trimmed with automated parameter detection using trimal v1.2 [86]. Phylogenetic relationships were inferred using IQTree2.0 [87]. The tree is rooted to the branch containing the outgroup $F$. venenatum (assembly $900,007,375.1$, a high quality genome of a close relative to $F$. poae [88]). Evolutionary histories were inferred using the Maximum Likelihood (ML) method and the best model was automatically determined per gene sequence using ModelFinder [89] as part of the IQTree 
v2.0.6 pipeline [87] utilizing partition modeling to allow genes to evolve under independent models [90]. The tree was calculated using an ultrafast bootstrapping value $(n=1000)$ and drawn to scale, with branch lengths measured in number of substitutions per site [91].

\section{APS1 gene survey}

To detect APS1 by PCR, primers were designed and used in a duplex reaction along with TEF1 $\alpha$ (Additional file 15). To eliminate the possibility of APS1 false negatives, TEF1 $\alpha$ was used as a positive control in the duplex reaction to ensure the DNA was amplifiable. All 184 isolates surveyed in this study were tested, as well as 9 Canadian and 10 international (total $n=203$ ). PCR was performed as described above except with an annealing temperature of $59^{\circ} \mathrm{C} .10 \mu \mathrm{L}$ was loaded on a $1 \%$ agarose gel.

\section{Abbreviations}

AC: Accessory chromosome; APS: Apicidin; BGC: Biosynthetic gene cluster; BUSCO: Benchmarked universal single copy orthologs; FHB: Fusarium head blight; NRPS: Non-ribosomal peptide synthetase; PKS: Polyketide synthase; RIP: Repeat-induced point mutation; STC: Sesquiterpene cyclase; TE: Transposable element; UPLC-HRMS: Ultra-high performance liquid chromatography coupled to high resolution mass spectrometry

\section{Supplementary Information}

The online version contains supplementary material available at https://doi. org/10.1186/s12864-021-07617-y.

Additional file 1 Summary of all F. poae isolates screened in this study. Data includes DAOMC accession numbers, crop pathology codes, locations, country of origin, host crop type, groupings inferred by analysis of TEF1- $a$, TR/1 and TR/8 genes, and results from the APS1 PCR survey.

Additional file $\mathbf{2}$ Jukes-Cantor/Neighbor-joining consensus tree of Clustal Omega alignment of concatenated TEF1a - TR/1 - TR/8 genomic sequences of 19 representative Ontario and Quebec isolates and one Belgian F. poae isolate (genome assembly LYXU01; Vanheule et al. 2016). The isolate name is followed by the number of isolates represented in brackets of the 193 Ontario and Quebec isolates surveyed. The TEF1a TR/1 and TR/8 genomic sequences of the 19 isolates have been deposited in Genbank as accession numbers MT571548-MT571566, MT578829MT578829-MT578847, and MT571567-MT571585, respectively.

Additional file 3 Summary of Illumina genome assembly statistics for 37 F. poae isolates profiled in this study.

Additional file $\mathbf{4}$ Genome assembly statistics for Fp157, assembly WOUF00000000.

Additional file 5 LASTZ comparison of F. poae Fp157 with F. poae 2516 (assembly GCA_001675295.1) and F. graminearum PH1 (assembly GCA_000240135.3).

Additional file 6. Media conditions used. Formulations for CYA, MMK2, S1M, S2M, YES, YESIO are listed.

Additional file 7. Matrix of averaged chemical phenotype data (all mass features included in analysis). Values represent detection frequencies averaged from 5 media conditions. Column headings (mass features) are in RT_ $m / z$ format, where RT refers to column retention time (minutes) and $m / z$ is the mass/charge ratio ( $<5 \mathrm{ppm}$ accuracy).

Additional file 8. Mirror plots of W-493 A (Top) and W-493 B (Bottom). Upper spectra in each mirror plot represent experimentally derived fragmentation patterns from F. poae extracts, bottom spectra are from GNPS libraries (spectral matches $<5 \mathrm{ppm}$ are coloured green).
Additional file 9. Total ion current and extract ion current chromatographs illustrating butenolide-associated peak (red arrows) eluting during start of run, in region normally sent to waste (blue bracket). Accompanying text explains putative butenolide annotation process.

Additional file 10. Mirror plots of apicidin $[\mathrm{M}+\mathrm{Na}]+($ top), apicidin $B$ $[\mathrm{M}+\mathrm{Na}]+$ (middle), and apicidin $\mathrm{C}[\mathrm{M}+\mathrm{Na}]+$ (bottom). Upper spectra in each mirror plot represent experimentally derived fragmentation patterns from F. poae extracts, bottom spectra are from GNPS libraries (spectral matches $<5 \mathrm{ppm}$ are coloured green).

Additional file 11. Mirror plot of $\mathrm{MS}^{2}$ spectra from features annotated as apicidin (APS) and APS-G. Overlaid chemical structures are proposed ions associated with specific fragments. Top: spectra of $\mathrm{m} / \mathrm{z}[\mathrm{M}+\mathrm{H}]+=$ 624.375 (APS structure top right). Spectra coloured purple are present in nearly all spectra associated with apicidins bearing O-methylated tryptophan moiety, including spectra annotated as APS, APS-B, APS-C, and APSD2. Structures outlined in purple are those proposed to contain indole substructures. Notably, nominal masses 130 and 170 have been associated with tryptophan fragmentation elsewhere ${ }^{5,6}$. Bottom: spectra from parent $\mathrm{m} / \mathrm{z}[\mathrm{M}+\mathrm{H}]+=555.354$ (annotated as APS-G, structure bottom right). Spectra coloured red are present uniquely from $\mathrm{MS}^{2}$ scans of this $m / z$, with corresponding proposed ion structures containing phenylalanine outlined in red. Spectra coloured green in bottom plot are $\mathrm{m} / \mathrm{z}$ within 5 ppm match for spectra on top plot.

Additional file 12. Expanded molecular network analysis of APS-like spectra with annotations overlaid. Labels are parent ion $\mathrm{m} / \mathrm{z}$. Node colours indicate ion identities (as identified by IIN module, unless the node outline is grey, in which case the ion was low intensity and didn't group with informative ions - annotation manual in this case). Blue nodes are $[\mathrm{M}+\mathrm{H}]+$, yellow nodes are $[\mathrm{M}+\mathrm{Na}]+$, green nodes are $[\mathrm{M}+\mathrm{NH} 4]+$, purple node is $[\mathrm{M}-\mathrm{H} 2 \mathrm{O}+\mathrm{H}]+$. Red bordered nodes were annotated as apicidins via in silico spectral analysis, green bordered nodes were annotated as apicidins via GNPS spectral matching (cos $>0.7)$. Blue lines indicate high spectral matching ( $\cos >0.7)$, red lines indicate ion identity matches (peak shape pearson correlation coefficients $>0.8$ ).

Additional file 13. Blastn comparison of FpAPS cluster to homologous clusters in F. sporotrichioides (PXOF00000000), F. langsethiae (JXCE00000000), and F. incarnatum (GQ331953).

Additional file 14. Duplex PCR screening for presence of APS1 in $F$. poae genomic DNA. Diagnostic bands for TEF1a and APS1 are indicated by arrows.

Additional file 15. List of primer sequences and amplicon sizes for TEF1a, TRI1, TRI8 and APS1.

Additional file 16. Detailed explanation of metabolomics data processing parameters, binary matrix conversion pipeline, qExactive instrument and data processing parameters and mass feature annotation pipeline details.

Additional file 17. Compressed folder containing alignments for each of the 4141 orthologs used in BUSCO analysis.

\section{Acknowledgements}

The authors sincerely thank Dr. Allen G. Xue and Yuanhong Chen (AAFCORDC) for supplying most of the F. poae isolates screened in this study, Kasia Dadej for Illumina sequencing services, and Dr. Rajagopal Subramanium for proof reading of the manuscript.

\section{Authors' contributions}

TW, LH and DO conceived and designed the study; TW performed metabolomics and genomics data analysis; LH assisted with genomics data analysis interpretation; DO assisted with metabolomics data analysis interpretation; HN performed genome assembly and annotation; AS processed extracts via UPLC-HRMS; AJ performed gDNA extractions, PCR amplifications, and Nanopore sequencing; AH cultured fungi in various media conditions and performed organic phase extractions; CB assisted with analysis of APS-G spectra and edited the manuscript; JD assisted with BUSCO data interpretation; TW, $\mathrm{LH}, \mathrm{HN}, \mathrm{AH}, \mathrm{AJ}, \mathrm{AS}, \mathrm{DO}$ wrote the manuscript; and all authors read, edited and approved the final manuscript. 


\section{Funding}

This research was funded by the AAFC research grant J-000048 (EmTOX: Research network on emerging mycotoxins of potential importance for Canadian agriculture and regulation of domestic commodities and international trade) and continued research funding support for the publication of this research is provided by the AAFC research grant J-002071 (The population structure of Fusarium pathogens of small grain cereals, their distribution and relationship to mycotoxins).

\section{Availability of data and materials}

A representative subset of Fusarium poae isolates used for genomic and metabolomics analysis in this study have been made publicly available by request and through standard permissions from the Canadian Collection of Fungal Cultures (Ottawa, ON, Canada; email: aafc.culturesfongiques-daomcfungalcultures.aac@canada.ca).

The annotated genome of Fp157 has been deposited with links to BioProject accession number PRJNA578270 in the NCBI BioProject database (https:// www.ncbi.nlm.nih.gov/bioproject/ PRJNA578270). Raw reads for Fp157 have also been uploaded to NCBI SRA, with accession number SRR13023856 for Illumina reads, and SRR13483968 for Nanopore reads. Sequences used for the BUSCO analysis have been uploaded as a compressed directory of fasta files in Additional file 17

\section{Declarations}

\section{Ethics approval and consent to participate}

Not applicable

\section{Consent for publication}

Not applicable.

\section{Competing interests}

The authors declare they have no competing interests.

\section{Author details}

${ }^{1}$ Agriculture and Agri-Food Canada, Ottawa Research and Development Centre, Ottawa, Canada. ${ }^{2}$ Department of Chemistry and Biomolecular Sciences, University of Ottawa, Ottawa, Canada.

Received: 25 November 2020 Accepted: 8 March 2021

Published online: 04 August 2021

\section{References}

1. Chakraborty S, Newton AC. Climate change, plant diseases and food security: an overview. Plant Pathol. 2011;60(1):2-14. https://doi.org/10.1111/ j.1365-3059.2010.02411.x.

2. Nielsen LK, Cook DJ, Edwards SG, Ray RV. The prevalence and impact of Fusarium head blight pathogens and mycotoxins on malting barley quality in UK. Int J Food Microbiol. 2014;179(100):38-49. https://doi.org/10.1016/j. ijfoodmicro.2014.03.023.

3. Vogelgsang S, Beyer M, Pasquali M, Jenny E, Musa T, Bucheli TD, et al. An eight-year survey of wheat shows distinctive effects of cropping factors on different Fusarium species and associated mycotoxins. Eur J Agron. 2019; 105:62-77. https://doi.org/10.1016/j.eja.2019.01.002

4. Xue AG, Chen Y, Seifert K, Guo W, Blackwell BA, Harris LJ, et al. Prevalence of Fusarium species causing head blight of spring wheat, barley and oat in Ontario during 2001-2017. Can J Plant Pathol 2019;0(00):1-11.

5. Xu XM, Parry DW, Nicholson P, Thomsett MA, Simpson D, Edwards SG, et al. Predominance and association of pathogenic fungi causing Fusarium ear blightin wheat in four European countries. Eur J Plant Pathol. 2005;112(2): 143-54. https://doi.org/10.1007/s10658-005-2446-7.

6. Valverde-Bogantes E, Bianchini A, Herr JR, Rose DJ, Wegulo SN, HallenAdams HE. Recent population changes of Fusarium head blight pathogens: drivers and implications. Can J Plant Pathol. 2019;42(3):315-29.

7. Schöneberg T, Jenny E, Wettstein FE, Bucheli TD, Mascher F, Bertossa M, et al. Occurrence of Fusarium species and mycotoxins in Swiss oats_impact of cropping factors. Eur J Agron. 2018;92:123-32. https://doi. org/10.1016/j.eja.2017.09.004

8. Stenglein SA. Fusarium poae: a pathogen that needs more attention. J Plant Pathol. 2009;91(1):25-36.
9. Thrane U, Adler A, Clasen PE, Galvano F, Langseth W, Lew H, et al. Diversity in metabolite production by Fusarium langsethiae, Fusarium poae, and Fusarium sporotrichioides. Int J Food Microbiol. 2004;95(3):257-66. https:// doi.org/10.1016/j.ijfoodmicro.2003.12.005.

10. Stenglein SA, Dinolfo MI, Barros G, Bongiorno F, Chulze SN, Moreno MV. Fusarium poae pathogenicity and mycotoxin accumulation on selected wheat and barley genotypes at a single location in Argentina. Plant Dis. 2014;98(12):1733-8. https://doi.org/10.1094/PDIS-02-14-0182-RE.

11. Rocha O, Ansari K, Doohan FM. Effects of trichothecene mycotoxins on eukaryotic cells: a review. Food Addit Contam. 2005;22(4):369-78. https:// doi.org/10.1080/02652030500058403.

12. EFSA. Scientific Opinion on the risks to human and animal health related to the presence of beauvericin and enniatins in food and feed. EFSA J. 2014; 12(8):3802.

13. Fraeyman S, Croubels S, Devreese M, Antonissen G. Emerging fusarium and alternaria mycotoxins: occurrence, toxicity and toxicokinetics. Toxins (Basel). 2017;9(7):1-26.

14. Tralamazza SM, Rocha LO, Oggenfuss U, Corrêa B, Croll D, Rose L. Complex evolutionary origins of specialized metabolite gene cluster diversity among the plant pathogenic Fungi of the Fusarium graminearum species complex. Rose L, editor. Genome Biol Evol. 2019;11(11):3106-22. https://doi.org/10.1 093/gbe/evz225

15. Hansen FT, Gardiner DM, Lysøe E, Fuertes PR, Tudzynski B, Wiemann P, et al. An update to polyketide synthase and non-ribosomal synthetase genes and nomenclature in Fusarium. Fungal Genet Biol. 2015;75:20-9. https://doi. org/10.1016/j.fgb.2014.12.004

16. Hoogendoorn K, Barra L, Waalwijk C, Dickschat JS, van der Lee TAJ, Medema $\mathrm{MH}$. Evolution and diversity of biosynthetic gene clusters in Fusarium. Front Microbiol. 2018;9(1158):1-12.

17. Vanheule A, Audenaert K, Warris S, van de Geest H, Schijlen E, Höfte M, et al. Living apart together: crosstalk between the core and supernumerary genomes in a fungal plant pathogen. BMC Genomics. 2016;17(1):670. https://doi.org/10.1186/s12864-016-2941-6

18. Hatta R, Ito K, Hosaki Y, Tanaka T, Tanaka A, Yamamoto M, et al. A conditionally dispensable chromosome controls host-specific pathogenicity in the fungal plant pathogen Alternaria alternata. Genetics. 2002;161(1):59-70.

19. Coleman JJ, Rounsley SD, Rodriguez-Carres M, Kuo A, Wasmann CC, Grimwood J, et al. The genome of Nectria haematococca: Contribution of supernumerary chromosomes to gene expansion. Madhani HD, editor. PLoS Genet. 2009;5(8):e1000618.

20. Ma L-JJ, Van Der Does HC, Borkovich KA, Coleman JJ, Daboussi M-JJ, Di Pietro A, et al. Comparative genomics reveals mobile pathogenicity chromosomes in Fusarium. Nature. 2010;464(7287):367-73. https://doi.org/1 0.1038 /nature08850

21. Bertazzoni S, Williams AH, Jones DA, Syme RA, Tan KC, Hane JK. Accessories make the outfit: accessory chromosomes and other dispensable DNA regions in plant-pathogenic fungi. Mol Plant-Microbe Interact. 2018;31(8): 779-88. https://doi.org/10.1094/MPMI-06-17-0135-FI.

22. Croll D, McDonald BA. The accessory genome as a cradle for adaptive evolution in pathogens. PLoS Pathog. 2012;8(4):e1002608.

23. Galazka JM, Freitag M. Variability of chromosome structure in pathogenic fungi-of "ends and odds". Curr Opin Microbiol. 2014;20:19-26. https://doi. org/10.1016/j.mib.2014.04.002.

24. Geiser DM, Jiménez-Gasco MDM, Kang S, Makalowska I, Veeraraghavan N, Ward TJ, et al. FUSARIUM-ID v. 1.0: a DNA sequence database for identifying Fusarium. Eur J Plant Pathol. 2004;1 10(5-6):473-9. https://doi.org/10.1023/B: EJPP.0000032386.75915.a0

25. McCormick SP, Harris LJ, Alexander NJ, Ouellet T, Saparno A, Allard S, et al. Tri1 in Fusarium graminearum Encodes a P450 Oxygenase. Appl Environ Microbiol. 2004;70(4):2044-51. https://doi.org/10.1128/AEM.70.4.2044-2051.2 004.

26. Varga E, Wiesenberger G, Hametner C, Ward TJ, Dong Y, Schöfbeck D, et al. New tricks of an old enemy: isolates of Fusarium graminearum produce a type a trichothecene mycotoxin. Environ Microbiol. 2015;17(8):2588-600. https://doi.org/10.1111/1462-2920.12718.

27. Alexander NJ, McCormick SP, Waalwijk C, van der Lee T, Proctor RH. The genetic basis for 3-ADON and 15-ADON trichothecene chemotypes in Fusarium. Fungal Genet Biol. 2011;48(5):485-95. https://doi.org/10.1016/j. fgb.2011.01.003.

28. Simão FA, Waterhouse RM, loannidis P, Kriventseva EV, Zdobnov EM. BUSCO: assessing genome assembly and annotation completeness with 
single-copy orthologs. Bioinformatics. 2015;31(19):3210-2. https://doi.org/1 0.1093/bioinformatics/btv351.

29. King R, Urban M, Hammond-Kosack MCU, Hassani-Pak K, Hammond-Kosack $\mathrm{KE}$. The completed genome sequence of the pathogenic ascomycete fungus Fusarium graminearum. BMC Genomics. 2015;16(1):544. https://doi. org/10.1186/s12864-015-1756-1.

30. Harris RS. Improved pairwise alignment of genomic DNA. PhD Thesis. State College: Pennsylvania State University; 2007.

31. Van Wyk S, Harrison CH, Wingfield BD, De Vos L, Van Der Merwe NA, Steenkamp ET. The RIPper, a web-based tool for genome-wide quantification of repeat-induced point (RIP) mutations. PeerJ. 2019;2019(7): e7447.

32. Tsuge T, Harimoto Y, Hanada K, Akagi Y, Kodama M, Akimitsu K, et al. Evolution of pathogenicity controlled by small, dispensable chromosomes in Alternaria alternata pathogens. Physiol Mol Plant Pathol. 2016;95:27-31. https://doi.org/10.1016/j.pmpp.2016.02.009.

33. Williams AH, Sharma M, Thatcher LF, Azam S, Hane JK, Sperschneider J, et al. Comparative genomics and prediction of conditionally dispensable sequences in legume-infecting Fusarium oxysporum formae speciales facilitates identification of candidate effectors. BMC Genomics. 2016;17(1):1-24.

34. Blin K, Shaw S, Steinke K, Villebro R, Ziemert N, Lee SY, et al. AntiSMASH 5.0: updates to the secondary metabolite genome mining pipeline. Nucleic Acids Res. 2019:47(W1):W81-7. https://doi.org/10.1093/nar/gkz310.

35. Brown DW, Proctor RH. Insights into natural products biosynthesis from analysis of 490 polyketide synthases from Fusarium. Fungal Genet Biol. 2016; 89:37-51. https://doi.org/10.1016/j.fgb.2016.01.008.

36. Jiao F, Kawakami A, Nakajima T. Effects of different carbon sources on trichothecene production and tri gene expression by Fusarium graminearum in liquid culture. FEMS Microbiol Lett. 2008;285(2):212-9. https://doi.org/10.1111/j.1574-6968.2008.01235.x

37. Peplow AW, Meek IB, Wiles MC, Phillips TD, Beremand MN. Tri16 is required for esterification of position C-8 during Trichothecene Mycotoxin production by Fusarium sporotrichioides. Appl Environ Microbiol. 2003; 69(10):5935-40. https://doi.org/10.1128/AEM.69.10.5935-5940.2003.

38. Nihei $\mathrm{K}$, Itoh $\mathrm{H}$, Hashimoto $\mathrm{K}$, Miyairi K, Okuno T. Antifungal cyclodepsipeptides, W493 a and B, from fusarium sp.: isolation and structural determination. Biosci Biotechnol Biochem. 1998;62(5):858-63. https://doi.org/10.1271/bbb.62.858.

39. Gelderblom WCA, Marasas WFO, Steyn PS, Thiel PG, Van Der Merwe KJ, Van Rooyen $\mathrm{PH}$, et al. Structure elucidation of fusarin $\mathrm{C}$, a mutagen produced by fusarium moniliforme. J Chem Soc Chem Commun. 1984;2:122-4.

40. Niehaus EM, Kleigrewe K, Wiemann P, Studt L, Sieber CMK, Connolly LR, et al. Genetic manipulation of the fusarium fujikuroi fusarin gene cluster yields insight into the complex regulation and fusarin biosynthetic pathway. Chem Biol. 2013;20(8):1055-66. https://doi.org/10.1016/j.chembiol.2013.07.004.

41. Thrane U. Development in the taxonomy of Fusarium species based on secondary metabolites. In: Summerell BA, Leslie JK, Backhouse D, Bryden WL, Burgess LW, editors. Fusarium: Paul E. Nelson Memorial Symposium. St. Paul: APS Press; 2001. p. 29-49.

42. Harris $\amalg$, Alexander NJ, Saparno A, Blackwell B, McCormick SP, Desjardins $A E$, et al. A novel gene cluster in Fusarium graminearum contains a gene that contributes to butenolide synthesis. Fungal Genet Biol. 2007;44(4):293306. https://doi.org/10.1016/j.fgb.2006.11.001

43. Studt L, Wiemann P, Kleigrewe K, Humpf HU, Tudzynski B. Biosynthesis of fusarubins accounts for pigmentation of fusarium Fujikuroi perithecia. Appl Environ Microbiol. 2012;78(12):4468-80. https://doi.org/10.1128/AEM. 00823-12.

44. McCormick SP, Alexander NJ, Harris LJ. CLM1 of fusarium graminearum encodes a longiborneol synthase required for culmorin production. Appl Environ Microbiol. 2010;76(1):136-41. https://doi.org/10.1128/AEM.02017-09.

45. Singh SB, Zink DL, Liesch JM, Mosley RT, Dombrowski AW, Bills GF, et al. Structure and chemistry of apicidins, a class of novel cyclic tetrapeptides without a terminal a-keto epoxide as inhibitors of histone deacetylase with potent antiprotozoal activities. J Org Chem. 2002;67(3):815-25. https://doi. org/10.1021/j0016088w.

46. Suciati GMJ. Isolation of the tetrapeptide apicidins $\mathrm{G}, \mathrm{H}$ and I from the fungus Fusarium semitectum. Nat Prod Commun. 2014;9(2):233-6.

47. Wang M, Carver JJ, Phelan W, Sanchez LM, Garg N, Peng Y, et al. Sharing and community curation of mass spectrometry data with global natural products social molecular networking. Nat Biotechnol. 2016;34(8):828-37. https://doi.org/10.1038/nbt.3597.
48. Schmid R, Petras D, Nothias L-F, Wang M, Aron AT, Jagels A, et al. Ion Identity Molecular Networking in the GNPS Environment. bioRxiv. 2020;2020. 05.11.088948.

49. Pluskal T, Castillo S, Villar-Briones A, Orešič M. MZmine 2: modular framework for processing, visualizing, and analyzing mass spectrometrybased molecular profile data. BMC Bioinformatics. 2010;11(1):395. https://doi. org/10.1186/1471-2105-11-395.

50. Dührkop K, Fleischauer M, Ludwig M, Aksenov AA, Melnik AV, Meusel M, et al. SIRIUS 4: a rapid tool for turning tandem mass spectra into metabolite structure information. Nat Methods. 2019;16(4):299-302. https://doi.org/10.1 038/s41592-019-0344-8.

51. Jin JM, Lee S, Lee J, Baek SR, Kim JC, Yun SH, et al. Functional characterization and manipulation of the apicidin biosynthetic pathway in Fusarium semitectum. Mol Microbiol. 2010;76(2):456-66. https://doi.org/1 0.1111/j.1365-2958.2010.07109.x.

52. Wicker T, Sabot F, Hua-Van A, Bennetzen JL, Capy P, Chalhoub B, et al. A unified classification system for eukaryotic transposable elements. Nature Reviews Genetics. 2007;8:973-82.

53. Karimi K, Arzanlou M, Pertot I. Weeds as Potential Inoculum Reservoir for Colletotrichum nymphaeae Causing Strawberry Anthracnose in Iran and Rep-PCR Fingerprinting as Useful Marker to Differentiate C. acutatum Complex on Strawberry. Front Microbiol. 2019;10(FEB):129.

54. Schmidt SM, Houterman PM, Schreiver I, Ma L, Amyotte S, Chellappan B, et al. MITEs in the promoters of effector genes allow prediction of novel virulence genes in Fusarium oxysporum. BMC Genomics. 2013;14(1):1-21.

55. Darkin-Rattray SJ, Gurnett AM, Myers RW, Dulski PM, Crumley TM, Allocco JJ, et al. Apicidin: a novel antiprotozoal agent that inhibits parasite histone deacetylase. Proc Natl Acad Sci U S A. 1996;93(23):13143-7. https://doi.org/1 0.1073/pnas.93.23.13143.

56. Vanheule A, De Boevre M, Moretti A, Scauflaire J, Munaut F, De Saeger S, et al. Genetic divergence and chemotype diversity in the fusarium head blight pathogen Fusarium poae. Toxins (Basel). 2017;9(9):1-19.

57. Vogelgsang S, Sulyok M, Hecker A, Jenny E, Krska R, Schuhmacher R, et al. Toxigenicity and pathogenicity of Fusarium poae and Fusarium avenaceum on wheat. Eur J Plant Pathol. 2008;122(2):265-76. https://doi.org/10.1007/s1 0658-008-9279-0.

58. Singh SB, Zink DL, Polishook JD, Dombrowski AW, Darkin-Rattray SJ, Schmatz DM, et al. Apicidins: novel cyclic tetrapeptides as coccidiostats and antimalarial agents from Fusarium pallidoroseum. Tetrahedron Lett. 1996; 37(45):8077-80. https://doi.org/10.1016/0040-4039(96)01844-8.

59. Villani A, Proctor RH, Kim H-S, Brown DW, Logrieco AF, Amatulli MT, et al. Variation in secondary metabolite production potential in the Fusarium incarnatum-equiseti species complex revealed by comparative analysis of 13 genomes. BMC Genomics. 2019;20(1):314. https://doi.org/10.1186/s12864-01 9-5567-7.

60. Xia JW, Sandoval-Denis M, Crous PW, Zhang XG, Lombard L. Numbers to names - restyling the Fusarium incarnatum-equiseti species complex. Persoonia Mol Phylogeny Evol Fungi. 2019;43(1):186-221. https://doi.org/1 0.3767/persoonia.2019.43.05.

61. Singh SB, Zink DL, Liesch JM, Dombrowski AW, Darkin-Rattray SJ, Schmatz DM, et al. Structure, histone deacetylase, and antiprotozoal activities of apicidins $B$ and $C$, congeners of apicidin with proline and valine substitutions. Org Lett. 2001;3(18):2815-8. https://doi.org/10.1021/ol016240g.

62. Lysøe E, Frandsen RJN, Divon HH, Terzi V, Orrù L, Lamontanara A, et al. Draft genome sequence and chemical profiling of Fusarium langsethiae, an emerging producer of type a trichothecenes. Int J Food Microbiol. 2016;221: 29-36. https://doi.org/10.1016/j.jifoodmicro.2016.01.008.

63. von Bargen KW, Niehaus E-M, Bergander K, Brun R, Tudzynski B, Humpf H-U. Structure elucidation and antimalarial activity of Apicidin F: an Apicidin-like compound produced by Fusarium fujikuroi. J Nat Prod. 2013;76(11):2136-40. https://doi.org/10.1021/np4006053.

64. Jin J, Baek SR, Lee KR, Lee J, Yun SH, Kang S, et al. Purification and phytotoxicity of apicidins produced by the Fusarium semitectum KCTC16676. Plant Pathol J. 2008;24(4):417-22. https//doi.org/10.5423/PPJ.2008.24.4.417.

65. Niehaus EM, Kim HK, Münsterkötter M, Janevska S, Arndt B, Kalinina SA, et al. Comparative genomics of geographically distant Fusarium fujikuroi isolates revealed two distinct pathotypes correlating with secondary metabolite profiles. PLoS Pathog. 2017:13(10):1-38.

66. Han JW, Ahn SH, Park SH, Wang SY, Bae GU, Seo DW, et al. Apicidin, a histone deacetylase inhibitor, inhibits proliferation of tumor cells via induction of p21WAF1/Cip1 and gelsolin. Cancer Res. 2000;60(21):6068-74. 
67. Ueda T, Takai N, Nishida M, Nasu K, Narahara H. Apicidin, a novel histone deacetylase inhibitor, has profound anti-growth activity in human endometrial and ovarian cancer cells. Int J Mol Med. 2007;19(2):301-8.

68. Walton JD. HC-toxin. Phytochemistry. 2006;67:1406-13.

69. Rajarammohan S, Paritosh K, Pental D, Kaur J. Comparative genomics of Alternaria species provides insights into the pathogenic lifestyle of Alternaria brassicae - a pathogen of the Brassicaceae family. BMC Genomics. 2019;20(1):1-13.

70. Wight WD, Labuda R, Walton JD. Conservation of the genes for HC-toxin biosynthesis in Alternaria jesenskae. BMC Microbiol. 2013;13(1):165. https:// doi.org/10.1186/1471-2180-13-165.

71. Waalwijk C, Taga M, Zheng SL, Proctor RH, Vaughan MM, O'Donnell K. Karyotype evolution in Fusarium. IMA Fungus. 2018;9(1):13-33. https://doi. org/10.5598/imafungus.2018.09.01.02.

72. Fekete $\mathrm{C}$, Hornok L. A repetitive DNA sequence associated with karyotype variability in Fusarium poae. Acta Phytopathol Entomol Hungarica. 1997; 32(1-2):29-38.

73. Mallebrera B, Prosperini A, Font G, Ruiz MJ. In vitro mechanisms of Beauvericin toxicity: a review. Food Chem Toxicol. 2018;111:537-45. https:// doi.org/10.1016/j.fct.2017.11.019.

74. Novak B, Rainer V, Sulyok M, Haltrich D, Schatzmayr G, Mayer E. Twentyeight fungal secondary metabolites detected in pig feed samples: their occurrence, relevance and cytotoxic effects in vitro. Toxins (Basel). 2019; 11(9):537. https://doi.org/10.3390/toxins11090537.

75. Wang M, Rogers S, Bittremieux W, Chen C, Dorrestein P, Schymanski E, et al. Interactive MS/MS Visualization with the Metabolomics Spectrum Resolver Web Service. bioRxiv. 2020;2020.05.09.086066.

76. Wandy J, Zhu Y, Van Der Hooft JJJ, Daly R, Barrett MP, Rogers S. Ms2lda.org: web-based topic modelling for substructure discovery in mass spectrometry. Bioinformatics. 2018;34(2):317-8. https://doi.org/10.1093/ bioinformatics/bt×582.

77. Bankevich A, Nurk S, Antipov D, Gurevich AA, Dvorkin M, Kulikov AS, et al. SPAdes: a new genome assembly algorithm and its applications to singlecell sequencing. J Comput Biol. 2012;19(5):455-77. https://doi.org/10.1089/ cmb.2012.0021.

78. Koren S, Walenz BP, Berlin K, Miller JR, Bergman NH, Phillippy AM. Canu: scalable and accurate long-read assembly via adaptive $\mathrm{K}$-mer weighting and repeat separation. Genome Res. 2017;27(5):722-36. https://doi.org/10.1101/ gr.215087.116.

79. Loman NJ, Quick J, Simpson JT. A complete bacterial genome assembled de novo using only nanopore sequencing data. Nat Methods. 2015;12(8): 733-5. https://doi.org/10.1038/nmeth.3444.

80. Li H, Durbin R. Fast and accurate short read alignment with burrowswheeler transform. Bioinformatics. 2009;25(14):1754-60. https://doi.org/10.1 093/bioinformatics/btp324.

81. Walker BJ, Abeel T, Shea T, Priest M, Abouelliel A, Sakthikumar S, et al. Pilon: An Integrated Tool for Comprehensive Microbial Variant Detection and Genome Assembly Improvement. Wang J, editor. PLoS One. 2014;9(11): e112963.

82. Haas BJ, Papanicolaou A, Yassour M, Grabherr M, Blood PD, Bowden J, et al. De novo transcript sequence reconstruction from RNA-seq using the trinity platform for reference generation and analysis. Nat Protoc. 2013;8(8):1494512. https://doi.org/10.1038/nprot.2013.084.

83. Love J, Palmer J, Stajich J, Esser T, Kastman E, Winter D. Funannotate v1.5.2; 2019.

84. Bao W, Kojima KK, Kohany O. Repbase update, a database of repetitive elements in eukaryotic genomes. Mob DNA. 2015;6(1):11. https://doi.org/1 0.1186/s13100-015-0041-9.

85. Katoh K, Standley DM. MAFFT multiple sequence alignment software version 7: improvements in performance and usability. Mol Biol Evol. 2013; 30(4):772-80. https://doi.org/10.1093/molbev/mst010.

86. Capella-Gutiérrez S, Silla-Martínez JM, Gabaldón T. trimAl: a tool for automated alignment trimming in large-scale phylogenetic analyses. Bioinformatics. 2009; 25(15):1972-3. https://doi.org/10.1093/bioinformatics/btp348.

87. Minh BQ, Schmidt HA, Chernomor O, Schrempf D, Woodhams MD, Von Haeseler A, et al. IQ-TREE 2: new models and efficient methods for phylogenetic inference in the genomic era. Mol Biol Evol. 2020;37(5):1530-4. https://doi.org/10.1093/molbev/msaa015.

88. King R, Brown NA, Urban M, Hammond-Kosack KE. Inter-genome comparison of the Quorn fungus Fusarium venenatum and the closely related plant infecting pathogen Fusarium graminearum. BMC Genomics. 2018;19(1):1-19.

89. Kalyaanamoorthy S, Minh BQ, Wong TKF, Von Haeseler A, Jermiin LS. ModelFinder: fast model selection for accurate phylogenetic estimates. Nat Methods. 2017;14(6):587-9. https://doi.org/10.1038/nmeth.4285.

90. Chernomor O, Von Haeseler A, Minh BQ. Terrace aware data structure for Phylogenomic inference from Supermatrices. Syst Biol. 2016;65(6):997-1008. https://doi.org/10.1093/sysbio/syw037.

91. Hoang DT, Chernomor O, von Haeseler A, Minh BQ, Vinh LS. UFBoot2: improving the ultrafast bootstrap approximation. Molecular biology and evolution. Mol Biol Evol. 2018;35(2):518-22. https://doi.org/10.1093/molbev/ $\mathrm{ms} \times 281$.

\section{Publisher's Note}

Springer Nature remains neutral with regard to jurisdictional claims in published maps and institutional affiliations.
Ready to submit your research? Choose BMC and benefit from:

- fast, convenient online submission

- thorough peer review by experienced researchers in your field

- rapid publication on acceptance

- support for research data, including large and complex data types

- gold Open Access which fosters wider collaboration and increased citations

- maximum visibility for your research: over $100 \mathrm{M}$ website views per year

At BMC, research is always in progress.

Learn more biomedcentral.com/submissions 